\title{
Single-user MIMO versus multi-user MIMO in distributed antenna systems with limited feedback
}

\author{
Stefan Schwarz ${ }^{1}$, Robert W Heath $\mathrm{Jr}^{2}$ and Markus Rupp ${ }^{1}$
}

\begin{abstract}
This article investigates the performance of cellular networks employing distributed antennas in addition to the central antennas of the base station. Distributed antennas are likely to be implemented using remote radio units, which is enabled by a low latency and high bandwidth dedicated link to the base station. This facilitates coherent transmission from potentially all available antennas at the same time. Such distributed antenna system (DAS) is an effective way to deal with path loss and large-scale fading in cellular systems. DAS can apply precoding across multiple transmission points to implement single-user MIMO (SU-MIMO) and multi-user MIMO (MU-MIMO) transmission. The throughput performance of various SU-MIMO and MU-MIMO transmission strategies is investigated in this article, employing a Long-Term evolution (LTE) standard compliant simulation framework. The previously theoretically established cell-capacity improvement of MU-MIMO in comparison to SU-MIMO in DASs is confirmed under the practical constraints imposed by the LTE standard, even under the assumption of imperfect channel state information (CSI) at the base station. Because practical systems will use quantized feedback, the performance of different CSI feedback algorithms for DASs is investigated. It is shown that significant gains in the CSI quantization accuracy and in the throughput of especially MU-MIMO systems can be achieved with relatively simple quantization codebook constructions that exploit the available temporal correlation and channel gain differences.
\end{abstract}

Keywords: Distributed antenna systems, Remote radio units, Channel state information estimation, Limited feedback communication, Quantized feedback, Single-user MIMO, Multi-user MIMO, LTE

\section{Introduction}

A distributed antenna system (DAS) is a cellular networking architecture in which several transmission points, controlled by a single central processing unit, are geographically distributed throughout the network. DASs make use of infrastructure like remote radio units (RRUs), with minimal intelligence of their own, to extend the base stations' antenna ports. RRUs are connected to the base station by a high-bandwidth low-latency dedicated connection that is non-interfering with the primary radio resource, e.g., a dedicated micro-wave link or a fiber link using radio-over-fiber technology [1]. DASs have been successful as a tool for coverage improvement in the

\footnotetext{
*Correspondence: sschwarz@nt.tuwien.ac.at

${ }^{1}$ Institute of Telecommunications, Vienna University of Technology, Vienna, Austria

Full list of author information is available at the end of the article
}

network, especially to mitigate shadowing effects and penetration losses [2]. Several publications have established the theoretical potential of DASs for improving the indoor coverage [3], reducing the outage probability [4], increasing the network capacity [5], providing a more uniform coverage of the served area [6] and improving the area spectral efficiency (ASE) of the system [6].

There are several ways to make use of distributed antennas. With single-user spatial multiplexing, a single user is served on a given time-frequency resource employing all available antenna elements of the DAS. Single-user MIMO (SU-MIMO) has been shown to yield higher sum rates in DASs that collocate about as many antennas per RRU as are available at the receiver side [7]. When multi-user MIMO (MU-MIMO) is employed, on the other hand, multiple users are served in parallel over the distributed antenna array, by separating

\section{是 Springer}

(C) 2013 Schwarz et al.; licensee Springer. This is an Open Access article distributed under the terms of the Creative Commons Attribution License (http://creativecommons.org/licenses/by/2.0), which permits unrestricted use, distribution, and reproduction in any medium, provided the original work is properly cited. 
their signals in the spatial domain. MU-MIMO transmission in DASs promises considerable performance gains, due to improved spatial multi-user diversity, compared to a system having collocated antennas only. Different multi-user multiplexing strategies for distributed antennas have been considered in the literature, embracing blanket transmission, i.e., using the same set of beamforming vectors at each RRU, and full MU-MIMO, where MUMIMO is applied across the entire distributed antenna array [6].

Less attention has been paid to limited feedback algorithms for DASs. An overview about the challenges of codebook design for channel state information (CSI) quantization in DASs and a possible solution were provided in [8]. Limited feedback single-user beamforming with distributed antennas was considered in [9]. MUMIMO in DASs with limited feedback and single antenna receivers is considered in [10], demonstrating substantial cell throughput gains with distributed antennas, provided sufficiently accurate channel state information at the transmitter (CSIT) is available. A differential CSI feedback algorithm was proposed in [10] that exploits spatial and temporal channel correlation to improve the quantization accuracy.

\subsection{Contributions}

In this article, the performance of different MIMO DAS strategies in cellular networks is evaluated under practical system constraints. Limited feedback algorithms for single-user and multi-user spatial multiplexing, optimized for DASs, are proposed. A 3GPP Long Term Evolution Advanced (LTE-A) standard compliant simulator, the Vienna LTE-A link level simulator $[11,12]$, is utilized to investigate the effectiveness of (SU-MIMO) and MU-MIMO in DASs, incorporating practical system parameters such as channel coding, finite modulation alphabets, and OFDM transmission. The achievable cellthroughput with perfect and quantized CSIT is explored, employing optimal and sub-optimal low-complexity transceiver structures.

New algorithms for limited feedback of CSI for MUMIMO DASs are proposed and compared, including memoryless and differential quantization algorithms. In frequency division duplexing systems, CSIT is usually acquired by means of limited capacity feedback channels from the users, thus requiring efficient CSI quantization algorithms. It is shown how side information on the largescale fading can be exploited to substantially improve the quantization accuracy. This is achieved by extending the MU-MIMO feedback algorithms of [10] to multiple receive antenna systems, employing single stream transmission per user with different receive antenna combining techniques. It is demonstrated that the largescale fading side information can also be utilized in
SU-MIMO systems to enable efficient transmit antenna subset selection.

All results in this article are derived considering the presence of out-of-cell interference. This is important because the distribution of RRUs over the cell area in DASs impacts the signal to interference and noise ratio (SINR) landscape. The overwhelming complexity of system-level simulations, caused by the fact that detailed knowledge about the physical layer is required for the considered precoding and equalization strategies, forces us to resort to a hybrid-link/system-level simulation method that was proposed in [10]. It combines detailed link-level simulations of a single cell with the abstracted interference model of [6]. With this simulation framework, the cell throughput of several DAS configurations is compared in terms of empirical cumulative density functions (ecdfs) of the transmission rate and ASE, with perfect and quantized CSIT. Furthermore, the performance of SU-MIMO and MU-MIMO systems is contrasted.

\subsection{Organization}

This article is organized as follows. In Section 2.1, the input-output relationship of a multi-cell, SU-MIMO, and MU-MIMO DAS is formulated, assuming linear precoding and equalization, and the post-equalization SINR is derived. Then, in Section 2.2 the transmission and reception strategies under consideration are reviewed. For SU-MIMO, singular value decomposition (SVD)-based precoding and equalization, and LTE-A compliant finite codebook-based precoding with zero forcing (ZF) equalization are considered. The MU-MIMO strategies use $\mathrm{ZF}$ beamforming at the base station and receive antenna combining based on multi-user eigenmode transmission (MET) and quantization-based combining $(\mathrm{QBC})$ at the receiver side. In Section 3.1, the SU-MIMO feedback algorithms are explained, and a transmit antenna subset selection algorithm is proposed. CSI feedback algorithms for MU-MIMO are proposed in Section 3.2. In Section 4.1, the employed simulation methodology is outlined, and in Section 4.2 the simulation settings are detailed. Simulation results with perfect CSIT, comparing several DAS configurations, are presented in Section 4.3. The performance of the proposed feedback algorithms is demonstrated in Section 4.4. Finally, conclusions are drawn in Section 5.

\subsection{Notation}

We use the following notation throughout this article: bold lowercase a is used to denote a column vector, bold uppercase A denotes a matrix. Non-bold letters $a$, $A$ denote scalars, and calligraphic letters $\mathcal{A}$ are used for sets. The magnitude of a scalar is $|a|,\|\mathbf{a}\|$ denotes the $l_{2}$ norm of a vector $\mathbf{a},\|\mathbf{A}\|$ is the Frobenius norm of a matrix $\mathbf{A}$, and the size of a set is given by $|\mathcal{A}|$. The 
inverse of a matrix is denoted $\mathbf{A}^{-1}$, the square root is $\mathbf{A}^{1 / 2}$, and the Moore-Penrose pseudo inverse is $\mathbf{A}^{\dagger}$. The notation $\mathbf{A} \in \mathbb{R}^{m \times n}\left(\mathbf{A} \in \mathbb{C}^{m \times n}\right)$ means that $\mathbf{A}$ is a real-valued (complex-valued) matrix of size $m$ rows times $n$ columns. Similarly, for length $n$ column vectors the notation $\mathbf{a} \in \mathbb{R}^{n}\left(\mathbf{a} \in \mathbb{C}^{n}\right)$ is used. We use the notation $\mathbf{a} \in \mathcal{N}(\mathbf{m}, \mathbf{R})$ to denote a complex-valued circularly symmetric Gaussian random vector $\mathbf{a}$, with mean $\mathbf{m}$ and covariance $\mathbf{R}$. The transpose of a matrix $\mathbf{A}$ is given by $\mathbf{A}^{\mathrm{T}}$, the conjugate-complex matrix is denoted $\mathbf{A}^{*}$ and the conjugate-transpose is written as $\mathbf{A}^{\mathrm{H}}$. The expected value of the vector a is denoted $\mathbb{E}\{\mathbf{a}\}$. The operator diag (a) creates a diagonal matrix, whose main diagonal entries equal a.

\section{System model}

In this section, the input-output relationship of the cellular network under consideration is presented and the post-equalization SINR experienced by a user is derived. Furthermore, the single-user and multi-user transmission and reception strategies under consideration are reviewed. Different strategies are employed depending on whether perfect or quantized CSIT is available. The derived system model is valid for a narrow-band, frequency flat channel model. However, the results can easily be extended to OFDM on a per-subcarrier basis with additional notation.

\subsection{Input-output relationship and post-equalization SINR} Consider a cellular network with $I+1$ cells. Each cell $i \in\{0, \ldots, I\}$ contains a central base station and $R_{i}$ RRUs. The central base station is equipped with an antenna array consisting of $N_{0, i}$ antenna elements. RRU $r \in\left\{1, \ldots, R_{i}\right\}$ has an antenna array comprising $N_{r, i}$ antenna elements. The total number of transmit antennas available in cell $i$ is denoted $N_{i}=\sum_{r=0}^{R_{i}} N_{r, i}$. An example network is shown in Figure 1.

Cell $i$ serves a total number of $U_{i}$ users. User $u \in$ $\mathcal{U}_{i}, \mathcal{U}_{i}=\left\{1, \ldots, \mathcal{U}_{i}\right\}$ is equipped with $M_{u, i}$ receive antennas. The base station contains a scheduling algorithm that decides for the set of users $\mathcal{S}_{i} \subseteq \mathcal{U}_{i}$ that is served in parallel over a given time-frequency resource, such as to maximize a prescribed utility function. In the simulations, the popular proportional fair scheduler is employed, which chooses the set of users to be served according to

$$
\mathcal{S}_{i}=\underset{\mathcal{S} \subseteq \mathcal{U}_{i}}{\operatorname{argmax}} \sum_{u \in \mathcal{S}} \frac{R_{u}}{T_{u}}, \text { subject to: }|\mathcal{S}| \leq C_{i} .
$$

Here, $R_{u}$ denotes the currently achievable throughput of user $u$, and $T_{u}$ denotes the average past throughput of

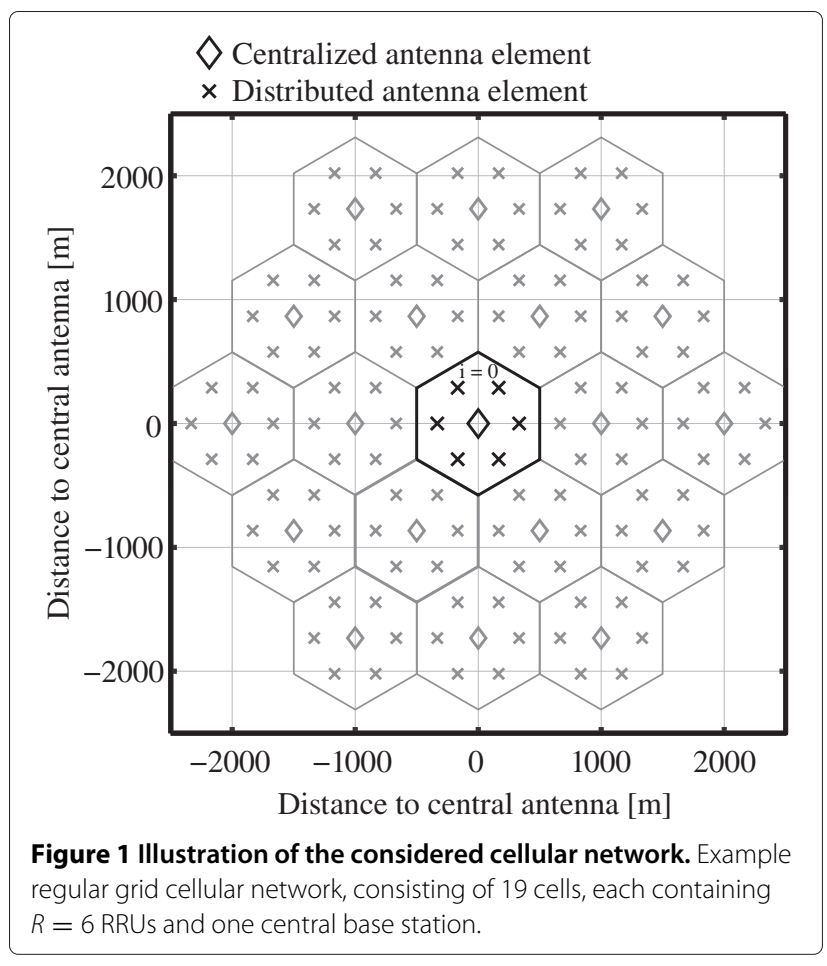

this user, obtained, e.g., with an exponential averaging filter. Under stationary assumptions it can be shown that this scheduling strategy maximizes the sum of logarithmic long-term average user rates [13]. $C_{i}$ is a constraint on the number of users that can be spatially multiplexed on a given time-frequency resource (e.g., an OFDM subcarrier). In the case of SU-MIMO only one user can be served at a given resource, $C_{i}=1$, while transmission to a maximum number of $C_{i}=N_{i}$ users in parallel is possible for MU-MIMO. The number of served users in cell $i$ is given by $S_{i}=\left|\mathcal{S}_{i}\right|$. To simplify notations, we assume that after scheduling the user set $\mathcal{U}_{i}$ is re-ordered such that the served users are indexed by $\left\{1, \ldots, S_{i}\right\}$.

The equivalent channel in the base-band between user $u$ in cell $i$ and RRU $r$ in cell $j$ is described by the complexvalued $M_{u, i} \times N_{r, j}$-dimensional channel matrix $\mathbf{H}_{u, i}^{(r, j)}$. The channels to the central base station and all $R_{j}$ RRUs are combined in the matrix

$$
\mathbf{H}_{u, i}^{(j)}=\left[\mathbf{H}_{u, i}^{(0, j)}, \ldots, \mathbf{H}_{u, i}^{\left(R_{j}, j\right)}\right] \in \mathbb{C}^{M_{u, i} \times N_{j}}
$$

To simplify notation, the superscript $j$ is omitted whenever in-cell channels, $i=j$, are considered.

The transmit symbol vector intended for user $u$ in cell $i$ is written as $\mathbf{x}_{u, i} \in \mathbb{C}^{\ell_{u, i}}$. Here, $\ell_{u, i}$ is the number of datastreams spatially multiplexed to user $u$. It is constrained by the number of receive antennas: $\ell_{u, i} \leq M_{u, i}$. The total number of streams of cell $i$ is denoted $\ell_{i}=\sum_{u=1}^{S_{i}} \ell_{u, i}$. 
To account for the maximum possible spatial multiplexing gain of cell $i$ the number of streams has to satisfy $\ell_{i} \leq N_{i}$. The average transmit symbol energy is assumed to be one: $\mathbb{E}\left\{\mathbf{x}_{u, i} \mathbf{x}_{u, i}^{\mathrm{H}}\right\}=\mathbf{I}$.

Prior to transmission over the wireless channel, the user symbol vector $\mathbf{x}_{u, i}$ is precoded with a precoding matrix $\mathbf{F}_{u, i} \in \mathbb{C}^{N_{i} \times \ell_{u, i}}$. The precoder maps the $\ell_{u, i}$-dimensional transmit symbol vector onto the $N_{i}$ transmit antennas. Note that the allocation of the available transmit power $P_{i}$ of cell $i$ among users and spatial streams is considered in the precoding matrices, as detailed in Section 2.2. The precoders are chosen such that $P_{i}$ is conserved, irrespective of the number of users, and spatial streams. The transmit symbols and precoding matrices of all served users are combined in the cell data vector and the cell precoding matrix

$$
\begin{aligned}
& \mathbf{x}_{i}=\left[\mathbf{x}_{1, i}^{\mathrm{T}}, \ldots, \mathbf{x}_{S_{i}, i}^{\mathrm{T}}\right]^{\mathrm{T}} \in \mathbb{C}^{\ell_{i}}, \\
& \mathbf{F}_{i}=\left[\mathbf{F}_{1, i}, \ldots, \mathbf{F}_{S_{i}, i}\right] \in \mathbb{C}^{N_{i} \times \ell_{i}} .
\end{aligned}
$$

We assume that users employ linear receive filters to equalize their respective channels. The $\ell_{u, i} \times M_{u, i}$ receive filtering matrix applied by user $u$, cell $i$, is denoted as $\mathbf{G}_{u, i}^{\mathrm{H}}$.

With this notation, the $\ell_{u, i}$-dimensional received signal vector of user $u$ in cell $i$ is

$$
\begin{gathered}
\mathbf{y}_{u, i}=\mathbf{G}_{u, i}^{\mathrm{H}} \mathbf{H}_{u, i} \mathbf{F}_{u, i} \mathbf{x}_{u, i}+\mathbf{G}_{u, i}^{\mathrm{H}} \mathbf{H}_{u, i} \sum_{k=1, k \neq u}^{S_{i}} \mathbf{F}_{k, i} \mathbf{x}_{k, i}+ \\
\underbrace{\mathbf{G}_{u, i}^{\mathrm{H}} \sum_{j=0, j \neq i}^{I} \mathbf{H}_{u, i}^{(j)} \mathbf{F}_{j} \mathbf{x}_{j}+\mathbf{G}_{u, i}^{\mathrm{H}} \mathbf{z}_{u, i}}_{\tilde{\mathbf{z}}_{u, i}},
\end{gathered}
$$

with $\mathbf{z}_{u, i} \in \mathcal{N}\left(\mathbf{0}, \sigma_{z}^{2} \mathbf{I}\right)$ denoting additive white Gaussian noise (AWGN). The effective noise vector $\tilde{\mathbf{z}}_{u, i}$ contains the sum of receiver noise and out-of-cell interference. The concatenation of the equalizer filter $\mathbf{G}_{u, i}^{\mathrm{H}}$ and the channel matrix $\mathbf{H}_{u, i}$ is called the effective channel of user $u$.

The instantaneous per-stream post-equalization SINR of stream $v \in\left\{1, \ldots, \ell_{u, i}\right\}$ equals

$$
\begin{aligned}
\beta_{v, u, i} & =\frac{\left|\mathbf{g}_{v, u, i}^{H} \mathbf{H}_{u, i} \mathbf{f}_{v, u, i}\right|^{2}}{Z+I_{\text {self }}+I_{\text {in }}+I_{\text {out }}}, \\
Z & =\sigma_{z}^{2}\left\|\mathbf{g}_{v, u, i}\right\|^{2}, \\
I_{\text {self }} & =\sum_{\mu=1, \mu \neq v}^{\ell_{u, i}}\left|\mathbf{g}_{v, u, i}^{H} \mathbf{H}_{u, i} \mathbf{f}_{\mu, u, i}\right|^{2}, \\
I_{\text {in }} & =\sum_{k=1, k \neq u}^{S_{i}}\left\|\mathbf{g}_{v, u, i}^{H} \mathbf{H}_{u, i} \mathbf{F}_{k, i}\right\|^{2}, \\
I_{\text {out }} & =\sum_{j=0, j \neq i}^{I}\left\|\mathbf{g}_{v, u, i}^{H} \mathbf{H}_{u, i}^{(j)} \mathbf{F}_{j}\right\|^{2} .
\end{aligned}
$$

Here, $\mathbf{g}_{v, u, i}$ and $\mathbf{f}_{v, u, i}$ denote the $v$ th column of $\mathbf{G}_{u, i}$ and $\mathbf{F}_{u, i}$, respectively, and $Z$ is the noise power after the receive filter. Note that $\mathbf{f}_{v, u, i}$ contains the transmit power allocation for stream $v$ of user $u$. The residual self-interference between the streams of a user after equalization is given by $I_{\text {self. }}$ The in-cell interference from other users that are served in parallel in the same cell is denoted $I_{\text {in }}$, and $I_{\text {out }}$ is the out-of-cell interference caused by users of other cells that operate at the same frequency. Note that depending on the transmission and reception strategy, some terms are equal to zero, e.g., for SU-MIMO there is no in-cell interference.

By distributing RRUs over the cell area, there will be path loss differences with respect to the spatially separated antenna arrays. To account for large-scale effects, the channel matrix is decomposed as

$$
\mathbf{H}_{u, i}^{(j)}=\overline{\mathbf{H}}_{u, i}^{(j)} \mathbf{C}_{u, i}^{(j)}{ }^{1 / 2}
$$

where the channel gain matrix $\mathbf{C}_{u, i}^{(j)}$ is diagonal and characterizes path loss and large-scale shadow fading effects experienced with respect to the different RRUs

$$
\mathbf{C}_{u, i}^{(j)}=\operatorname{diag}\left(\left[\gamma_{u, i}^{(1, j)}, \gamma_{u, i}^{(2, j)}, \ldots, \gamma_{u, i}^{\left(N_{j}, j\right)}\right]\right)
$$

and $\gamma_{u, i}^{(k, j)}$ denotes the large-scale channel gain between all receive antennas of user $u$ in cell $i$ and the transmit antenna $k$ in cell $j$. Note that if multiple antenna elements are collocated at one RRU, then the corresponding largescale channel gains are equal. Furthermore, the channel gain matrix can incorporate the transmit antenna gains if directional antennas are considered. The matrix $\overline{\mathbf{H}}_{u, i}^{(j)}$ accounts for small-scale fading effects.

\subsection{Considered transmission strategies}

In this section, the SU-MIMO and MU-MIMO precoding and equalization strategies considered in this study are briefly introduced and reviewed. The respective matrices $\mathbf{F}_{u, i}$ and $\mathbf{G}_{u, i}$ are assumed to be computed from perfect or estimated in-cell channel knowledge only, without explicit consideration of out-of-cell interference, to avoid the necessity of feeding back out-of-cell CSI. The transmitter and receiver thus treat out-of-cell interference as additional Gaussian noise, denoted as effective noise. Note that Gaussianity of the out-of-cell interference may not be fulfilled, e.g., if there are only a few dominant interferers. Then better performance can be achieved with receivers that estimate the interference statistics.

The investigated transceiver architectures are designed such as to avoid in-cell interference between users and spatial streams. With perfect channel knowledge at the base station and users, this can be achieved by transmitting over the eigenmodes of the channel(s). With imperfect CSIT, on the other hand, we consider unitary 
precoding at the base station combined with ZF equalization at the user in case of SU-MIMO. For MU-MIMO, we combine ZF beamforming at the base station with linear antenna combining at the users, to achieve similar complexity to SU-MIMO.

\subsubsection{SU-MIMO}

In SU-MIMO transmission only one user is served on a given time-frequency resource within a cell, possibly over multiple streams. With the simplifying assumption that out-of-cell interference is treated as additional Gaussian noise by the base station and users, the channel model reduces to a (distributed) point-to-point MIMO channel $\left(I_{\text {in }}=0\right)$. The capacity of the point-to-point AWGN MIMO channel with perfect CSI at the transmitter and receiver is achieved by SVD precoding and reception along with power loading across the eigenmodes of the channel [14]. While perfect CSI is unrealistic, it still provides a good benchmark for comparing the performance of limited feedback techniques. It has been shown that a substantial part of the capacity can be achieved with only a few bits of feedback allowed from the receiver to the transmitter [15]. Thus, limited feedback-based SU-MIMO precoding has been incorporated in 3GPP Long Term Evolution (LTE). The finite set of precoders defined in the LTE-A specifications [16] for limited feedback precoding is employed in our simulations.

SVD-based precoding and equalization Assuming SVD-based MIMO transmission and reception, the precoders and equalizers are obtained from the SVD of the wireless channel matrix. To shorten notation, we consider the case $M_{u, i} \leq N_{i}$, which is more relevant for DASs. In this case, the compact-form SVD can be written as

$$
\begin{aligned}
\mathbf{H}_{u, i} & =\mathbf{U}_{u, i} \mathbf{S}_{u, i} \mathbf{V}_{u, i}^{\mathrm{H}}, \\
\mathbf{S}_{u, i} & =\operatorname{diag}\left(\left[\sigma_{u, i}^{(1)}, \ldots, \sigma_{u, i}^{\left(\ell_{\max }\right)}\right]\right),
\end{aligned}
$$

where $\sigma_{u, i}^{(k)}$ is the $k$ th largest singular value of $\mathbf{H}_{u, i}$, and $\mathbf{U}_{u, i} \in \mathbb{C}^{M_{u, i} \times M_{u, i}}, \mathbf{V}_{u, i} \in \mathbb{C}^{N_{i} \times M_{u, i}}$ are (semi-) unitary matrices. $^{\text {a }}$ Assuming a full-rank channel, the maximum number of streams is $\ell_{\max }=M_{u, i}$. Setting the precoder as $\mathbf{F}_{u, i}=\mathbf{V}_{u, i} \mathbf{P}_{u, i}^{1 / 2}$, with $\mathbf{P}_{u, i}$ being a diagonal power loading matrix, and the equalizer as $\mathbf{G}_{u, i}=\mathbf{U}_{u, i}$, the effective channel decomposes into parallel non-interfering SISO AWGN channels. To achieve the highest transmission rate, the diagonal elements of $\mathbf{P}_{u, i}$ have to be set according to the water filling power allocation over the squared singular values normalized by the effective noise variance [14]. The effective noise variance $\tilde{\sigma}_{z}^{2}$ is calculated as

$$
\tilde{\sigma}_{z}^{2}=\sigma_{z}^{2}+\frac{1}{M_{u, i}} \sum_{j=0, j \neq i}^{I}\left\|\mathbf{H}_{u, i}^{(j)} \mathbf{F}_{j}\right\|^{2}
$$

assuming equal and spatially uncorrelated out-of-cell interference power on all receive antennas. Furthermore, the power loading matrix has to fulfill

$$
\operatorname{trace}\left(\mathbf{P}_{u, i}\right)=P_{i},
$$

to satisfy the total power constraint of base station $i$. Note that this strategy is not capacity achieving for nonGaussian or colored out-of-cell interference.

LTE codebook-based precoding with ZF equalization 3GPP decided in the LTE and LTE-A specifications [16,17] on a limited feedback precoding strategy that is based on a finite set/codebook of scaled semi-unitary precoders. The user searches this codebook for the best precoder based on its channel estimate and feeds back the index of the chosen precoder to the base station. The LTE Rel. 8 codebook for up to four transmit antennas is designed such as to enable computationally efficient selection criteria at the users, by exploiting properties of the employed Householder matrices [18]. LTE-A adds an eight-transmit antenna codebook, that is generated from the multiplication of two precoding matrices, a wideband and a subband precoder, which enables efficient feedback by exploiting spatial correlation of the MIMO channel [19]. Transmission rank adaptation is supported in LTE and LTE-A, which allows the number of streams to be selected dynamically. The precoders from the LTE codebooks assign equal powers to all streams.

We denote the precoder codebook for $\ell$ streams and $N_{i}$ transmit antennas as

$$
\mathcal{Q}_{\ell}^{\left(N_{i}\right)} \subset\left\{\mathbf{Q} \in \mathbb{C}^{N_{i} \times \ell} \mid \mathbf{Q}^{\mathrm{H}} \mathbf{Q}=\frac{1}{\ell} \mathbf{I}_{\ell}\right\} .
$$

If user $u$ is selected for transmission by the base station $i$, the base station applies the precoder

$$
\mathbf{F}_{u, i}=\sqrt{P_{i}} \mathbf{Q}_{u, i}, \mathbf{Q}_{u, i} \in \mathcal{Q}_{\ell_{u, i}}^{\left(N_{i}\right)}
$$

according to the precoder feedback $\mathbf{Q}_{u, i}$ of user $u$; see Section 3.1 for details. The precoder distributes the data for transmission onto the antenna elements all RRUs.

To separate the spatial data streams at the receiver, a linear equalizer filter can be used. In our simulations, ZF equalization at the users is considered. In this case, the receive filtering matrix is

$$
\mathbf{G}_{u, i}=\left(\left(\mathbf{H}_{u, i} \mathbf{F}_{u, i}\right)^{\dagger}\right)^{H}=\mathbf{H}_{u, i} \mathbf{F}_{u, i}\left(\mathbf{F}_{u, i}^{\mathrm{H}} \mathbf{H}_{u, i}^{\mathrm{H}} \mathbf{H}_{u, i} \mathbf{F}_{u, i}\right)^{-1} .
$$

LTE codebook-based precoding with transmit antenna subset selection The LTE precoders distribute the available transmit power uniformly over all antennas that are employed for transmission. Without transmit antenna subset selection, this means that the antenna elements of 
all RRUs transmit with equal power. When path loss differences between the distributed antenna arrays become significant, it is beneficial to concentrate the transmit power on those antenna arrays that experience good channel quality, thus employing only a subset of the antennas for transmission. To transmit the data from $a \leq N_{i}$ antennas, the LTE codebook $\mathcal{Q}_{\ell}^{(a)}$ is employed to distribute the signal onto the activated antennas, while the other antenna elements are deactivated. Note that LTE codebooks are only defined for $a$ being a power of 2 . In Section 3.1.3, we propose an efficient antenna subset selection algorithm. As an alternative, power loading over transmit antennas can be employed [20], but this requires accurate knowledge of the channel gain at the transmitter and thus increases the CSI feedback overhead significantly.

\section{$2.2 .2 \mathrm{MU}-\mathrm{MIMO}$}

With MU-MIMO, multiple users are served in parallel over a given time-frequency resource by means of spatial multiplexing. While in SU-MIMO the multiplexing gain is limited by the minimum of the number of transmit and receive antennas, in MU-MIMO the multiplexing gain scales with the number of transmit antennas, provided there are enough users in the cell. Although multiple streams per user are possible in MU-MIMO, it has been shown that single stream transmission per user is asymptotically optimal in the number of user $U_{i}$ [21] and that for finite number of users mostly only one stream is activated per selected user $i \in \mathcal{S}_{i}$ [22]. Thus, in this article only single stream transmission per user is considered for simplicity, employing ZF beamforming. The receive matrix $\mathbf{G}_{u, i}$ then reduces to the vector $\mathbf{g}_{u, i}$. To calculate the $\mathrm{ZF}$ precoder, the users have to feedback their effective channel vector, i.e., the channel generated by the concatenation of the wireless channel matrix $\mathbf{H}_{u, i}$ and the receive filter $\mathbf{g}_{u, i}$. In a limited feedback system, the effective channel is quantized to limit the amount of feedback bits required. Two methods for obtaining the antenna combining vector $\mathbf{g}_{u, i}$ are considered, namely QBC [23] and MET [22]. The goal of MET is to choose the combining weights such as to maximize the gain of the effective user channel, neglecting interference between users. QBC targets a minimal quantization error, thereby reducing the interference between users for the price of a reduced effective channel gain compared to MET. It is shown that both methods can be combined to allow a trade-off between these two extreme targets.

ZF beamforming with multi-user eigenmode transmission In single stream multi-user eigenmode transmission, the receive antenna combining weights $\mathbf{g}_{u, i}$ are computed such that the effective channel is in the direction of the maximum singular value of the channel matrix $\mathbf{H}_{u, i}$ [22], thereby maximizing the effective channel gain

$$
\begin{aligned}
\mathbf{g}_{u, i}= & \underset{\mathbf{g} \in \mathbb{C}^{M_{u, i}}}{\operatorname{argmax}}\left\|\mathbf{g}^{\mathrm{H}} \mathbf{H}_{u, i}\right\|^{2}, \\
& \text { subject to: }\|\mathbf{g}\|^{2}=1 .
\end{aligned}
$$

The solution to this problem is obtained from the SVD of the channel matrix Equation (9)

$$
\begin{aligned}
\mathbf{g}_{u, i} & =\mathbf{u}_{u, i}^{(\max )}, \\
\mathbf{h}_{u, i} & =\mathbf{H}_{u, i}^{\mathrm{H}} \mathbf{g}_{u, i}=\mathbf{v}_{u, i}^{(\max )} \sigma_{u, i}^{(\max )} .
\end{aligned}
$$

The vectors $\mathbf{u}_{u, i}^{(\max )}$ and $\mathbf{v}_{u, i}^{(\max )}$ denote the left and right singular vectors corresponding to the maximum singular value $\sigma_{u, i}^{(\max )}$ of $\mathbf{H}_{u, i}$. The direction of the effective MISO channel $\mathbf{h}_{u, i}$ is determined by $\mathbf{v}_{u, i}^{(\max )}$. The input-output relationship (5) simplifies to

$$
y_{u, i}=\mathbf{h}_{u, i}^{\mathrm{H}} \mathbf{f}_{u, i} x_{u, i}+\mathbf{h}_{u, i}^{\mathrm{H}} \sum_{k=1, k \neq u}^{S_{i}} \mathbf{f}_{k, i} x_{k, i}+\tilde{z}_{u, i},
$$

where the precoding matrices $\mathbf{F}_{k, i}$ are replaced with precoding vectors $\mathbf{f}_{k, i}$, and $\tilde{z}_{u, i}$ denotes the sum of out-of-cell interference and receiver noise.

Assuming that the base station $i$ has perfect knowledge of $\mathbf{h}_{u, i}, \forall u$, ZF beamforming can be applied across the set of scheduled users $\mathcal{S}_{i}$ to cancel all in-cell interference $I_{\text {in }}$. With imperfect CSIT, perfect interference cancellation is not possible by means of precoding and thus residual incell interference is unavoidable. The ZF beamformer can be obtained from knowledge of the normalized effective channel vectors (channel directions), defined as

$$
\tilde{\mathbf{h}}_{u, i}=\frac{\mathbf{h}_{u, i}}{\left\|\mathbf{h}_{u, i}\right\|} .
$$

Combining the effective channel directions of the selected users in the effective cell channel direction matrix

$$
\tilde{\mathbf{H}}_{i}=\left[\tilde{\mathbf{h}}_{1, i}, \ldots, \tilde{\mathbf{h}}_{S_{i}, i}\right]^{\mathrm{H}},
$$

the ZF precoder is

$$
\begin{aligned}
\mathbf{F}_{i} & =\tilde{\mathbf{H}}_{i}^{\mathrm{H}}\left(\tilde{\mathbf{H}}_{i} \tilde{\mathbf{H}}_{i}^{\mathrm{H}}\right)^{-1} \operatorname{diag}\left(\mathbf{p}_{i}\right)^{1 / 2}=\mathbf{W}_{i} \operatorname{diag}\left(\mathbf{p}_{i}\right)^{1 / 2}, \\
\mathbf{p}_{i} & =\left[p_{1, i}, \ldots, p_{S_{i}, i}\right], \quad p_{k, i}=\frac{P_{i}}{S_{i}\left\|\mathbf{w}_{k, i}\right\|^{2}} .
\end{aligned}
$$

It is thus given by the right pseudo-inverse of $\tilde{\mathbf{H}}_{i}$ multiplied by a diagonal power loading matrix. The power loading matrix ensures equal transmit powers across users and a sum transmit power of $P_{i}$.

ZF beamforming with quantization-based receiver combining With imperfect channel knowledge at the base station, interference between users cannot be entirely 
pre-canceled. When the base station employs ZF beamforming calculated from quantized CSIT, the residual in-cell interference between users depends on the accuracy of the quantized channel direction, specifically on the subspace distance between the channel direction and the quantized channel direction, see Section 3.2.2. The quantized effective user channel direction, available at the base station, is denoted as $\mathbf{q}_{u, i}$.

The idea behind $\mathrm{QBC}$ is to choose the antenna combining weights such as to generate an effective channel that can be quantized with minimal quantization error, given the quantization codebook $\mathcal{Q} \subseteq \mathbb{C}^{N_{i}},\|\mathbf{q}\|=1, \forall \mathbf{q} \in \mathcal{Q}$. In essence, the algorithm jointly selects the quantization vector and calculates the antenna combining weights such as to minimize the subspace distance (chordal distance)

$$
\left\{\mathbf{g}_{u, i}, \mathbf{q}_{u, i}\right\}=\underset{\mathbf{g} \in \mathbb{C}^{M_{u, i}, \mathbf{q} \in \mathcal{Q},\|\mathbf{g}\|=1}}{\operatorname{argmin}} 1-\left.\left|\frac{\mathbf{g}^{\mathrm{H}} \mathbf{H}_{u, i}}{\left\|\mathbf{g}^{\mathrm{H}} \mathbf{H}_{u, i}\right\|}\right|^{2}\right|^{2} .
$$

The algorithmic steps to achieve this goal are summarized below; for a detailed treatment see $[23]^{\mathrm{b}}$.

1. Determine an orthonormal basis for the space spanned by the rows of $\mathbf{H}_{u, i}$. The SVD of the conjugate-transpose channel matrix

$$
\mathbf{H}_{u, i}^{\mathrm{H}}=\mathbf{V}_{u, i} \mathbf{S}_{u, i} \mathbf{U}_{u, i}^{\mathrm{H}} \text {. }
$$

allows to define a basis $\mathbf{B}_{u, i} \in \mathbb{C}^{N_{i} \times M_{u, i}}$ by the left singular vectors corresponding to non-zero singular values of $\mathbf{H}_{u, i}^{\mathrm{H}}$.

2. Find the quantization vector that minimizes the subspace distance to the space spanned by $\mathbf{B}_{u, i}$ :

$$
\mathbf{q}_{u, i}=\underset{\mathbf{q} \in \mathcal{Q}}{\operatorname{argmin} 1-\left\|\mathbf{B}_{u, i}^{\mathrm{H}} \mathbf{q}\right\|^{2} .}
$$

3. The antenna combining weights are then given by

$$
\begin{aligned}
\mathbf{s}_{u, i} & =\frac{\mathbf{B}_{u, i} \mathbf{B}_{u, i}^{\mathrm{H}} \mathbf{q}_{u, i}}{\left\|\mathbf{B}_{u, i} \mathbf{B}_{u, i}^{\mathrm{H}} \mathbf{q}_{u, i}\right\|}, \\
\mathbf{g}_{u, i} & =\frac{\left(\mathbf{H}_{u, i}^{\mathrm{H}}\right)^{\dagger} \mathbf{s}_{u, i}}{\left\|\left(\mathbf{H}_{u, i}^{\mathrm{H}}\right)^{\dagger} \mathbf{s}_{u, i}\right\|} .
\end{aligned}
$$

A quantization function $\Omega^{(\mathrm{QBC})}\left(\mathbf{H}_{u, i}\right): \mathbb{C}^{M_{u, i} \times N_{i}} \rightarrow \mathcal{Q}$ is used to describe the combined action of steps 1 and 2 of the algorithm, hence $\mathbf{q}_{u, i}=\Omega^{(\mathrm{QBC})}\left(\mathbf{H}_{u, i}\right)$.

The quantized channel knowledge $\mathbf{q}_{u, i}$ is used at the base station to compute the ZF precoder, by replacing the cell channel matrix $\mathbf{H}_{i}$ in (21) with its quantized version

$$
\begin{aligned}
\mathbf{Q}_{i} & =\left[\mathbf{q}_{1, i}, \ldots, \mathbf{q}_{S_{i}, i}\right]^{\mathrm{H}}, \\
\mathbf{F}_{i} & =\mathbf{Q}_{i}^{\mathrm{H}}\left(\mathbf{Q}_{i} \mathbf{Q}_{i}^{\mathrm{H}}\right)^{-1} \operatorname{diag}\left(\mathbf{p}_{i}\right)^{1 / 2}=\mathbf{W}_{i} \operatorname{diag}\left(\mathbf{p}_{i}\right)^{1 / 2} .
\end{aligned}
$$

\section{Dominant subspace quantization-based receiver com-} bining $\mathrm{MET}$ and $\mathrm{QBC}$ are two extreme cases of antenna combining techniques. The former method does only consider the effective channel gain, irrespective of the achieved CSI quantization error, while the latter algorithm focuses on the quantization error, regardless of the gain of the effective channel obtained.

It is possible to trade-off between these two extremes, by restricting the optimization in quantization-based combining to the $k$-dimensional dominant subspace of the rows of $\mathbf{H}_{u, i}$. This can be achieved by replacing the matrix $\mathbf{B}_{u, i}$ in (25) and (26) with the $N_{i} \times k$ dimensional matrix $\mathbf{B}_{k, u, i}$, consisting of the left singular vectors corresponding to the $k$ largest singular values of $\mathbf{H}_{u, i}^{\mathrm{H}}$ obtained from (24). In that way, QBC is confined to calculate the antenna combining weights such that the effective channel lies in the $k$-dimensional dominant subspace of the channel, while simultaneously minimizing the quantization error. Thus, the optimization problem (23) is replaced with

$$
\begin{aligned}
\left\{\mathbf{g}_{u, i}, \mathbf{q}_{u, i}\right\}=\underset{\mathbf{g} \in \mathbb{C}^{M_{u, i}, \mathbf{q} \in \mathcal{Q},\|\mathbf{g}\|=1}}{\operatorname{argmin}} 1-\left|\frac{\mathbf{g}^{\mathrm{H}} \mathbf{H}_{u, i}}{\left\|\mathbf{g}^{\mathrm{H}} \mathbf{H}_{u, i}\right\|}\right|^{2}, \\
\text { subject to: } \mathbf{H}_{u, i}^{\mathrm{H}} \mathbf{g} \in \operatorname{span}\left(\mathbf{B}_{k, u, i}\right)
\end{aligned}
$$

where $\operatorname{span}\left(\mathbf{B}_{k, u, i}\right)$ denotes the space spanned by the columns of the matrix $\mathbf{B}_{k, u, i}$.

For illustration, consider the extreme case of an unrestricted codebook $\mathcal{Q}=\mathbb{C}^{N_{i}},\|\mathbf{q}\|=1, \forall \mathbf{q} \in \mathcal{Q}$ and $k=1$. In that case, it can easily be verified that the $\mathrm{QBC}$ solution coincides with the one obtained from multi-user eigenmode transmission.

\section{Limited feedback algorithms}

The transceiver architectures presented in Section 2.2 have in common that they require CSI at the transmitter to enable precoding and transmission rate adaptation. In practice, CSIT is obtained by means of feedback from the users, over dedicated limited capacity feedback links. In commercial cellular systems, the limited feedback operation consists of two parts. One part provides information about the channel quality experienced by a user. It is required for multi-user scheduling and transmission rate adaptation. An estimate of the expected SINR of a user is employed for that purpose in this study. Typically coarse quantization of the channel quality is sufficient to achieve a throughput performance that is close to unquantized feedback, because practical communication system like LTE provide only a limited set of modulation and coding schemes (MCSs) from which to choose during rate adaptation (see, e.g., [24]).

The second feedback part provides information about the channel matrix itself to the base station, which is required for precoding purposes. In the case of SU-MIMO transmission, this information is indirectly provided by signaling the preferred precoder from 
the finite precoder set defined in (12). Only a few bits of information can lead to substantial throughput gains [15]. With MU-MIMO, on the other hand, the effective channel vector is quantized and fed back by the users. The proposed quantization algorithms build upon the concepts of Grassmannian beamforming [25]. The achieved user throughput directly depends on the CSI accuracy, because it determines the residual interference between users. Consequently, accurate quantization is important for such MU-MIMO systems.

\subsection{SU-MIMO feedback}

The SU-MIMO feedback algorithms presented in this section leverage the ideas of finite codebook based limited feedback precoding, as introduced in Section 2.2.1. Our proposal of [24] is employed, which targets OFDMbased communication systems like LTE. As our focus in this study is on frequency-flat systems, we specialize the algorithms below to this case. For more general case of frequency-selective channels, the interested reader is referred to [24]. We also propose an efficient transmit antenna subset selection algorithm in Section 3.1.3. In DASs, antenna subset selection can significantly improve the transmission rate, as demonstrated in Section 4. The reason is that with distributed antennas, the path loss differences between transmission points become more significant. Thus, it is beneficial to concentrate the transmit power only on those antennas that experience good channel quality, instead of equally distributing it over all antennas.

\subsubsection{Estimation of the achievable spectral efficiency}

The basic idea of the considered SU-MIMO feedback algorithms is to let each user $u$ select that precoder from the set of codebooks $\left\{\mathcal{Q}_{1}^{\left(N_{i}\right)}, \ldots, \mathcal{Q}_{\ell_{\max }^{\left(N_{i}\right)}}\right\}$, that maximizes the achievable spectral efficiency of the system. The codebooks $\mathcal{Q}_{\ell}^{\left(N_{i}\right)}$ are defined in (12); $\ell_{\max }=\min \left(M_{u, i}, N_{i}\right)$ is the maximum transmission rank. To uniquely identify the precoder at the base station, two feedback values are employed. The codebook $\mathcal{Q}_{\ell}^{\left(N_{i}\right)}$ from which the preferred precoder is chosen, i.e., the index $\ell$, is signaled with the rank indicator (RI). The index of the selected precoder in the codebook $\mathcal{Q}_{\ell}^{\left(N_{i}\right)}$ is fed back with the precoding matrix indicator (PMI). Given this choice, the quality of the resulting channel is quantified with the post-equalization SINR. This value is quantized and fed back employing the channel quality indicator (CQI).

The spectral efficiency obtained with the rank $\ell$ pre$\operatorname{coder} \mathbf{Q}^{(\ell)} \in \mathcal{Q}_{\ell}^{\left(N_{i}\right)}$ is

$$
I\left(\mathbf{Q}^{(\ell)}\right)=\sum_{\nu=1}^{\ell} \varphi\left(\beta_{\nu, u, i}\right) .
$$

It is calculated as the sum spectral efficiency over the number of streams $\ell$. The function $\varphi(\cdot)$ maps the SINR $\beta_{v, u, i}$ to the corresponding spectral efficiency. Employing the ZF receiver of (14), $\beta_{v, u, i}$ defined in (6) simplifies to

$$
\beta_{v, u, i}=P_{i} \frac{\left|\mathbf{g}_{v, u, i}^{H} \mathbf{H}_{u, i} \mathbf{q}_{v}^{(\ell)}\right|^{2}}{Z+I_{\text {out }}},
$$

where $\mathbf{q}_{v}^{(\ell)}$ denotes the $v$ th column of $\mathbf{Q}^{(\ell)}$. Thus, only outof-cell interference and noise are disturbing the singleuser transmission. It is assumed that the user is able to estimate the power $\tilde{\sigma}_{z}^{2}$ of this effective noise.

In contrast to other work, e.g., [26], the bit interleaved coded modulation (BICM) capacity [27] is employed for $\varphi(\cdot)$ in our algorithms to estimate the spectral efficiency, instead of the AWGN channel capacity. The BICM capacity includes the effects of employing a finite modulation alphabet, which causes a transmission rate saturation at high SINR (e.g., 6 bits/cu for 64 QAM). Although this is not so important for frequency flat systems, it has a strong impact on the accuracy of the spectral efficiency estimation for LTE in frequency selective systems; see [24] for details.

\subsubsection{Calculation of the SU-MIMO CSI feedback}

The optimal precoder and the preferred number of streams are jointly obtained by maximizing the sum spectral efficiency (31)

$$
\left\{\mathbf{Q}_{u, i}, \ell_{u, i}\right\}=\underset{\mathbf{Q}^{(\ell)} \in \mathcal{Q}_{\ell}, \ell \in\left\{1, \ldots, \ell_{\max }\right\}}{\operatorname{argmax}} I\left(\mathbf{Q}^{(\ell)}\right) .
$$

It is assumed that the base station employs these choices during transmission, provided the user is scheduled.

The channel quality measure is obtained by mapping the sum spectral efficiency $I\left(\mathbf{Q}_{u, i}\right)$ back to the corresponding SINR value, via the inverse $\varphi^{-1}(\cdot)$ of the BICM capacity

$$
\bar{\beta}_{u, i}=\varphi^{-1}\left(I\left(\mathbf{Q}_{u, i}\right)\right) .
$$

This approach corresponds to mutual information effective SINR mapping, which is well known from link- to system-level abstraction [28]. The value $\bar{\beta}_{u, i}$ is denoted as equivalent SISO AWGN signal to noise ratio (SNR), because it corresponds to the SNR of an equivalent SISO AWGN channel that achieves the same spectral efficiency as the considered MIMO system. The CQI is calculated by quantizing the equivalent SNR

$$
\mathrm{CQI}_{u, i}=\Psi\left(\bar{\beta}_{u, i}\right),
$$

where $\Psi: \mathbb{R} \rightarrow \mathcal{C} \subseteq \mathbb{R}$ denotes the CQI quantizer. In our simulations, we employ a look-up table for $\Psi$ which is calibrated such as to achieve a block-error ratio of less than or equal to 0.1, given the LTE MCSs. The look-up table was determined by extensive link-level simulations. Following LTE conventions, this feedback mode is denoted as closed loop spatial multiplexing (CLSM). 


\subsubsection{CLSM with transmit antenna subset selection}

To enable efficient transmit antenna subset selection, with minimal extra CSI feedback overhead, it is assumed that the in-cell channel gain matrix $\mathbf{C}_{u, i}$ from (8) is known by both, the base station and the user. This is a reasonable, often made assumption in DASs, because the large-scale statistics change only very slowly over time and frequency, and can thus be learned either from the uplink, provided the duplex distance is not too large, or via a very low rate feedback link (e.g., during connection setup).

To determine the preferred antenna subset, the user can calculate and maximize the average spectral efficiency (31) for all possible antenna subset choices. This amounts to

$$
\sum_{a=1}^{N_{i}}\left(\begin{array}{c}
N_{i} \\
a
\end{array}\right)=2^{N_{i}}-1
$$

possibilities, and thus to an additional feedback overhead of $N_{i}$ bits.

To reduce the amount of feedback, the LTE-specific constraint that only one, two, four, or eight transmit antennas can be employed is exploited. This restricts $a$ in (36) to the set $\mathcal{A}=\{1,2,4,8\}$, and thus reduces the feedback overhead from eight to seven bits. In addition, the knowledge of $\mathbf{C}_{u, i}$ is used in that only the antenna subsets with the largest channel gains are tested. Thus, e.g., if $a=4$, the average spectral efficiency is only calculated for the four strongest distributed antennas. In that way, the additional CSI overhead is reduced to $\log _{2} \mathcal{A}=2$ bits.

\subsection{MU-MIMO feedback}

Providing useful CSI feedback for ZF multi-user beamforming systems is a more intricate challenge than in the SU-MIMO system. One reason is that in contrast to single-user transmission the SINR can only be bounded in MU-MIMO, because a user does neither know the employed precoder nor the number of users scheduled in parallel ahead of transmission. The corresponding bound and channel quality feedback algorithm are derived in Section 3.2.2.

Furthermore, accurate knowledge about the effective user channel directions is important at the base station, to minimize residual in-cell interference. Three possible quantization strategies are proposed in Section 3.2.3: memoryless quantization with and without consideration of the channel gain differences between different RRUs, and differential quantization. In memoryless quantization, the effective channel direction at each transmission time interval (TTI) is quantized independently of previous time instants. The differential quantizer, on the other hand, exploits temporal channel correlation and quantizes only the difference between channel directions at consecutive time instants. The proposed algorithms are an extension of the CSI quantizers of [10] to multiple receive antennas.

It is possible to extend the considered quantization algorithms to frequency-selective OFDM systems. This can be done efficiently, by providing CSI feedback as proposed in this section for a subset of the OFDM subcarriers, and interpolating in-between these CSI pilot-positions at the base station $[29,30]$.

\subsubsection{MU-MIMO DAS with limited feedback}

With limited feedback, the base station uses quantized channel state information to compute the transmit beamforming vectors as in (29). The quantized effective channel direction of user $u$ in cell $i$ is denoted $\mathbf{q}_{u, i}$. In case the receive antenna combiner is determined independently of the CSI quantization (e.g., with MET), the quantized effective channel direction is obtained as

$$
\mathbf{q}_{u, i}=\Omega\left(\tilde{\mathbf{h}}_{u, i}\right)
$$

where $\Omega: \mathbb{C}^{N_{i}} \rightarrow \mathcal{Q}$ is the quantization function, which is specified below. In the case of quantization-based combining a more general quantization function, which acts on the channel matrix itself, is used; see Section 2.2.2. The vector $\tilde{\mathbf{h}}_{u, i}$ is the effective channel direction, given by

$$
\tilde{\mathbf{h}}_{u, i}=\frac{\mathbf{h}_{u, i}}{\left\|\mathbf{h}_{u, i}\right\|}=\frac{\mathbf{H}_{u, i}^{\mathrm{H}} \mathbf{g}_{u, i}}{\left\|\mathbf{H}_{u, i}^{\mathrm{H}} \mathbf{g}_{u, i}\right\|}=\frac{\mathbf{C}_{u, i}^{1 / 2} \overline{\mathbf{H}}_{u, i}^{\mathrm{H}} \mathbf{g}_{u, i}}{\left\|\mathbf{C}_{u, i}^{1 / 2} \overline{\mathbf{H}}_{u, i}^{\mathrm{H}} \mathbf{g}_{u, i}\right\|}
$$

with $\mathbf{g}_{u, i}$ being the antenna combining vector. Note the different usage of the codebook $\mathcal{Q}$ in the SU-MIMO and MU-MIMO cases. Here, $\mathcal{Q}$ is only used for quantization; the actual precoder $\mathbf{F}_{i}$ is computed from the quantized channel directions. In SU-MIMO, the entries defined in $\mathcal{Q}_{l}^{\left(N_{i}\right)}$ are directly employed as precoders.

The channel direction quantization error $\mathbf{e}_{u, i}$ is defined by the relationship [31]

$$
\tilde{\mathbf{h}}_{u, i}=\mathbf{q}_{u, i}\left(\mathbf{q}_{u, i}^{\mathrm{H}} \tilde{\mathbf{h}}_{u, i}\right)+\mathbf{e}_{u, i} .
$$

The quantization error is thus equal to the component of $\tilde{\mathbf{h}}_{u, i}$ in the null-space of the quantized channel direction $\mathbf{q}_{u, i}$. The squared magnitude of $\mathbf{e}_{u, i}$ is equal to the squared chordal distance between $\tilde{\mathbf{h}}_{u, i}$ and $\mathbf{q}_{u, i}$

$$
\begin{aligned}
d_{c}^{2}\left(\mathbf{q}_{u, i}, \tilde{\mathbf{h}}_{u, i}\right) & =\left\|\mathbf{e}_{u, i}\right\|^{2}=1-\left|\mathbf{q}_{u, i}^{\mathrm{H}} \tilde{\mathbf{h}}_{u, i}\right|^{2} \\
& =1-\cos ^{2}\left(\phi_{u, i}\right)=\sin ^{2}\left(\phi_{u, i}\right) .
\end{aligned}
$$

Here, $\phi_{u, i}$ denotes the principle angle between the onedimensional subspaces defined by $\tilde{\mathbf{h}}_{u, i}$ and $\mathbf{q}_{u, i}$, respectively, which can be obtained from the relationship

$$
\mathbf{q}_{u, i}^{\mathrm{H}} \tilde{\mathbf{h}}_{u, i}=e^{j \beta_{u, i}} \cos \left(\phi_{u, i}\right), \beta_{u, i} \in \mathbb{R} .
$$

Given the set $\mathcal{S}_{i}$ of scheduled users, the base station $i$ calculates the $Z F$ precoder according to (29). Using this 
notation, the input-output relationship for ZF beamforming with quantized CSIT can be written as

$$
\begin{aligned}
y_{u, i}= & \sqrt{p_{u, i}}\left\|\mathbf{h}_{u, i}\right\| \tilde{\mathbf{h}}_{u, i}^{\mathrm{H}} \mathbf{w}_{u, i} x_{u, i}+\left\|\mathbf{h}_{u, i}\right\| \\
& \times \sum_{k=1, k \neq u}^{S_{i}} \sqrt{p_{k, i}} \tilde{\mathbf{h}}_{u, i}^{\mathrm{H}} \mathbf{w}_{k, i} x_{k, i}+\tilde{z}_{u, i} \\
= & \sqrt{p_{u, i}}\left\|\mathbf{h}_{u, i}\right\|\left(e^{-j \beta_{u, i}} \cos \left(\phi_{u, i}\right) \mathbf{q}_{u, i}^{\mathrm{H}}+\mathbf{e}_{u, i}^{\mathrm{H}}\right) \mathbf{w}_{u, i} x_{u, i} \\
& +\left\|\mathbf{h}_{u, i}\right\| \sum_{k=1, k \neq u}^{S_{i}} \sqrt{p_{k, i}}\left(e^{-j \beta_{u, i}} \cos \left(\phi_{u, i}\right) \mathbf{q}_{u, i}^{\mathrm{H}}+\mathbf{e}_{u, i}^{\mathrm{H}}\right) \\
& \times \mathbf{w}_{k, i} x_{k, i}+\tilde{z}_{u, i} .
\end{aligned}
$$

Here, $\mathbf{w}_{u, i}$ is the $u$ th column of the matrix $\mathbf{W}_{i}$ defined in (29). This relationship can further be simplified, by realizing that $\mathbf{q}_{u, i}^{\mathrm{H}} \mathbf{w}_{u, i}=1$ and $\mathbf{q}_{u, i}^{\mathrm{H}} \mathbf{w}_{k, i}=0, \forall u \neq k$ by definition of the ZF beamformer. Thus, we get

$$
\begin{aligned}
y_{u, i}= & \sqrt{p_{u, i}}\left\|\mathbf{h}_{u, i}\right\|\left(e^{-j \beta_{u, i}} \cos \left(\phi_{u, i}\right)+\mathbf{e}_{u, i}^{\mathrm{H}} \mathbf{w}_{u, i}\right) x_{u, i} \\
& +\left\|\mathbf{h}_{u, i}\right\| \sum_{k=1, k \neq u}^{S_{i}} \sqrt{p_{k, i}} \mathbf{e}_{u, i}^{\mathrm{H}} \mathbf{w}_{k, i} x_{k, i}+\tilde{z}_{u, i} .
\end{aligned}
$$

\subsubsection{Channel quality feedback}

The purpose of channel quality feedback is to inform the base station about the SINR of the channel defined in (43), for scheduling and transmission rate adaptation. Here a causality problem occurs, because the schedule $\mathcal{S}_{i}$ depends on the CQI feedback, and the CQI feedback depends on the schedule. In our algorithm, this problem is resolved by basing the scheduling decision not on the exact SINR, but on a lower bound. The exact SINR of user $u$ in cell $i$ is

$$
\beta_{u, i}=\frac{p_{u, i}\left\|\mathbf{h}_{u, i}\right\|^{2}\left|e^{-j \beta_{u, i}} \cos \left(\phi_{u, i}\right)+\mathbf{e}_{u, i}^{\mathrm{H}} \mathbf{w}_{u, i}\right|^{2}}{\tilde{\sigma}_{z}^{2}+\left\|\mathbf{h}_{u, i}\right\|^{2} \sin ^{2}\left(\phi_{u, i}\right) \sum_{k \neq u} p_{k, i}\left|\tilde{\mathbf{e}}_{u, i}^{\mathrm{H}} \mathbf{w}_{k, i}\right|^{2}},
$$

where $\tilde{\sigma}_{z}^{2}$ denotes the power of the effective noise, and $\tilde{\mathbf{e}}_{u, i}$ is the unit norm error vector defined by

$$
\mathbf{e}_{u, i}=\sin \left(\phi_{u, i}\right) \tilde{\mathbf{e}}_{u, i} \text {. }
$$

In [31], the expected value of this SINR is lower bounded, for the case that random vector quantization is employed as channel direction quantization strategy (see below), and that a centralized system, i.e., one without distributed antennas, is employed

$$
\mathbb{E}\left(\beta_{u, i}\right) \geq \frac{\frac{P}{N_{i}}\left\|\mathbf{h}_{u, i}\right\|^{2} \cos ^{2}\left(\phi_{u, i}\right)}{\tilde{\sigma}_{z}^{2}+\frac{P}{N_{i}}\left\|\mathbf{h}_{u, i}\right\|^{2} \sin ^{2}\left(\phi_{u, i}\right)}=\beta_{u, i}^{(\mathrm{LB})} .
$$

Further assumptions made in [31] are that $N_{i}$ users are served in parallel, and that the quantization error- and precoding vectors are isotropically distributed. In DASs, the assumption of isotropic quantization error- and precoding vectors might not be valid anymore, because in contrast to centralized systems the channel vector is not isotropic either. Still, a lower bound that takes into account the effects of channel gain differences between antenna elements does not yet exist, and thus we resort to that bound as initial CQI employed for scheduling

$$
\mathrm{CQI}_{u, i}=\Psi\left(\beta_{u, i}^{(\mathrm{LB})}\right) \text {. }
$$

For rate adaptation, it turned out during simulations, that this SINR bound is not sufficiently accurate, especially in connection with the more advanced channel direction quantizers presented below. It is thus assumed, that the scheduled users provide a CQI correction as soon as the schedule is fixed and thus the SINR can be estimated accurately from (44). Note that in LTE a single CQI correction is sufficient for the total system bandwidth, because the same transmission rate is employed over all assigned subcarriers. This is in contrast to the initial CQI which should be frequency selective, to enable exploitation of frequency diversity during multi-user scheduling. In our simulations, we assume the CQI correction to be available instantaneously, i.e., without a feedback delay. In practice, a minimum delay of at least one TTI cannot be avoided, thus possibly leading to transmission outages, due to outdated CQI corrections. Such transmission errors can partly be compensated by the hybrid automatic repeat request protocol of LTE [32]. Further investigations with respect to feedback delays are still required to determine their impact on the system performance.

\subsubsection{Channel direction feedback}

Here, different possibilities for the channel direction quantizer function $\Omega$ are investigated. In this study, only joint quantization schemes are considered, where all the entries of $\tilde{\mathbf{h}}_{u, i}$ are quantized at once, using a single vector codebook. With limited feedback, it is important to quantize only the CSI that is required at the base station, to minimize feedback overhead. In the case of ZF precoding, suppose that the cell channel direction matrix $\tilde{\mathbf{H}}_{i}$ of (20) is pre-multiplied by a matrix $\Lambda=\operatorname{diag}\left(\exp \left(j \theta_{1}\right), \ldots, \exp \left(j \theta_{S_{i}}\right)\right)$. Then notice that $\left(\Lambda \tilde{\mathbf{H}}_{i}\right)^{\dagger}=\tilde{\mathbf{H}}_{i}^{\dagger} \Lambda^{\dagger}$. Thus, if the cell precoder $\mathbf{F}_{i}$ is calculated from a phase shifted version of the channel directions, the effective channel $\mathbf{H}_{i} \mathbf{F}_{i}=\Lambda^{\dagger}$ is still diagonal and in-cell interference is canceled. The resulting phase shift can easily be compensated by the user, and therefore the phase of $\tilde{\mathbf{h}}_{u, i}$ is irrelevant. Consequently, the transmitter requires only knowledge of the subspace defined by $\tilde{\mathbf{h}}_{u, i}$ to calculate the ZF precoder, and therefore Grassmannian subspace quantization can be employed for channel direction quantization [33]. The relevant Grassmannian manifold is the manifold of one-dimensional subspaces in 
the $N_{i}$-dimensional complex Euclidean space, denoted as $\mathcal{G}\left(N_{i}, 1\right)$.

The idea of Grassmannian quantization is to select the quantized channel direction $\mathbf{q}_{u, i}$ from a suitable quantization codebook $\mathcal{Q} \subset \mathcal{G}\left(N_{i}, 1\right)$ such as to minimize the subspace distance between the one-dimensional subspaces defined by $\mathbf{q}_{u, i}$ and the effective channel direction $\tilde{\mathbf{h}}_{u, i}$, respectively. The subspace distance is equal to the chordal distance defined in (40), and thus the quantization function $\Omega$ is

$$
\begin{aligned}
\mathbf{q}_{u, i} & =\Omega\left(\tilde{\mathbf{h}}_{u, i}\right):=\underset{\mathbf{q} \in \mathcal{Q}}{\operatorname{argmin}} d_{c}\left(\mathbf{q}, \tilde{\mathbf{h}}_{u, i}\right), \\
d_{c, \min } & =d_{c}\left(\mathbf{q}_{u, i}, \tilde{\mathbf{h}}_{u, i}\right) .
\end{aligned}
$$

This quantization function can be employed whenever the receive antenna combiner is obtained independently from the quantized channel direction, thus, e.g., with MET. In case of quantization-based combining, the quantization error is determined by the subspace distance between the one-dimensional subspace defined by $\mathbf{q}_{u, i}$ and the $M_{u, i}$-dimensional subspace spanned by rows of the channel matrix. Using the notation from Section 2.2.2, the corresponding quantization function is obtained as in (25)

$$
\begin{aligned}
& \mathbf{q}_{u, i}=\Omega^{(\mathrm{QBC})}\left(\mathbf{H}_{u, i}\right):=d_{c}\left(\mathbf{q}, \mathbf{H}_{u, i}\right)=1-\left\|\mathbf{B}_{u, i}^{\mathrm{H}} \mathbf{q}\right\|^{2}, \\
& d_{c, \text { min }}=d_{c}\left(\mathbf{q}_{u, i}, \mathbf{H}_{u, i}\right),
\end{aligned}
$$

with $\mathbf{B}_{u, i}$ being an orthonormal basis for the rows of $\mathbf{H}_{u, i}$; see Section 2.2.2.

In the following, some useful examples of quantization codebook constructions are considered.

\subsubsection{Memoryless quantization}

Memoryless quantization means that the channel direction at each TTI is quantized in isolation, using the same quantization codebook every time. The choice of this codebook determines the quantization mean squared error (MSE)

$$
\operatorname{MSE}=\mathbb{E}\left(d_{c, \min }^{2}\right) .
$$

In case the effective channel direction is isotropically distributed, the codebook design approach advocated in [25], which maximizes the minimum subspace distance between all vectors of the quantization codebook (codevectors), is a good choice. The resulting codebook constructions are called Grassmannian line packings, and the optimum codebook is essentially uniform on the Grassmannian manifold in terms of the subspace distance. Unfortunately, except for special cases, it is hard to find good codebooks. Thus, in our simulations, random isotropically distributed codebooks are employed, which achieve close performance to Grassmannian line packings in many cases [15]. The simulation results are averaged over codebook realizations. The resulting channel direction quantization algorithm is named random channel direction quantization (RCDQ), and the codebook is denoted $\mathcal{Q}^{(\mathrm{ISO})} \subset \mathcal{G}\left(N_{i}, 1\right)$. It is also known as random vector quantization, and often utilized to obtain theoretical performance results in limited feedback beamforming systems, e.g., $[34,35]$.

\subsubsection{Memoryless quantization with correlation}

The Grassmannian line packings introduced above assume that the effective channel direction to be quantized is isotropically distributed on the Grassmannian manifold $\mathcal{G}\left(N_{i}, 1\right)$.

When the user has multiple receive antennas, the effective channel vector $\mathbf{h}_{u, i}$ is given by a linear combination of the columns of $\mathbf{H}_{u, i}^{\mathrm{H}}$, see (38). Thus, the distribution of the effective channel depends on the considered antenna combining strategy. Love and Heath [36] propose a heuristic quantization codebook for limited feedback singleuser MISO beamforming systems with spatially correlated channel vectors, which effectively "colors" a Grassmannian codebook, by multiplying the codevectors with the channel correlation matrix. This technique was denoted as correlated Grassmannian beamforming in [36]. We take this approach one step further here to support multiple receive antenna systems. For simplicity, the small-scale fading channel matrix $\overline{\mathbf{H}}_{u, i}^{\mathrm{H}}$ is assumed spatially uncorrelated, but a possible correlation can straightforwardly be incorporated in the proposed codebook construction as well; thus for Rayleigh fading the elements of $\overline{\mathbf{H}}_{u, i}^{\mathrm{H}}$ are independent identically distributed (i.i.d.) $\mathcal{N}(0,1)$. In this case, the spatial channel correlation is only determined by the antenna combining technique and the channel gain matrix $\mathbf{C}_{u, i}$. Knowledge of $\mathbf{C}_{u, i}$ is assumed at both, the base station and the user.

As a motivating example one of the simplest antenna combining strategies, namely receive antenna selection, is considered. It activates only one antenna $v$ at a time by setting the corresponding combiner weight equal to one, and deactivates all other antennas by setting their weights equal to zero: $\mathbf{g}_{u, i}=\boldsymbol{\delta}_{v}$. Consequently, the effective channel is given by

$$
\mathbf{h}_{u, i}=\mathbf{C}_{u, i}^{1 / 2} \overline{\mathbf{H}}_{u, i}^{\mathrm{H}} \boldsymbol{\delta}_{v}=\mathbf{C}_{u, i}^{1 / 2} \overline{\mathbf{h}}_{v, u, i}
$$

with $\overline{\mathbf{h}}_{v, u, i}$ being the $v$ th column of $\overline{\mathbf{H}}_{u, i}^{\mathrm{H}}$. The effective channel is thus distributed according to $\mathcal{N}\left(\mathbf{0}, \mathbf{C}_{u, i}\right)$. Following [36], given the codebook $\mathcal{Q}^{(\mathrm{ISO})}$, the codebook for receive antenna selection is obtained as

$$
\mathcal{Q}^{(\mathrm{AS})}=\left\{\frac{\mathbf{C}_{u, i}^{1 / 2} \mathbf{q}_{l}^{(\mathrm{ISO})}}{\left\|\mathbf{C}_{u, i}^{1 / 2} \mathbf{q}_{l}^{(\mathrm{ISO})}\right\|} \mid \mathbf{q}_{l}^{(\mathrm{ISO})} \in \mathcal{Q}^{(\mathrm{ISO})}\right\} .
$$

Thus, with an isotropically distributed codebook $\mathcal{Q}^{(\mathrm{ISO})}$, the distribution of the directions defined in $\mathcal{Q}^{(\mathrm{AS})}$ matches the distribution of the effective channel direction. 
A generalization of the antenna selection idea is considered in quantization-based combining. Here, the user is not only allowed to select one of the one-dimensional subspaces specified by the channels to the $M_{u, i}$ individual receive antennas, but the effective channel can be chosen freely in the $M_{u, i}$-dimensional subspace spanned by all these channels. Because each of the channels specifying the subspace is $\mathcal{N}\left(\mathbf{0}, \mathbf{C}_{u, i}\right)$, the same codebook construction as in antenna selection is still valid

$$
\mathcal{Q}^{(\mathrm{QBC})}=\mathcal{Q}^{(\mathrm{AS})} .
$$

If multi-user eigenmode transmission is employed, the effective channel direction is equal to the maximum right singular vector of the channel matrix $\mathbf{H}_{u, i}$. Thus, the distribution of the channel direction is determined by the distribution of the largest singular vector. We are not aware of closed form expression for this distribution that would allow to generate statistically matched random quantization codebooks. It is therefore proposed to generate codevectors by manipulating random realizations of an iid complex Gaussian matrix

$$
\mathcal{Q}^{(\mathrm{MET})}=\left\{\mathbf{v}^{(\mathrm{max})}\left(\mathbf{Q}^{(\mathrm{iid})} \mathbf{C}_{u, i}^{1 / 2}\right) \mid \mathbf{Q}^{(\mathrm{iid})} \sim \mathcal{N}(\mathbf{0}, \mathbf{I})\right\},
$$

with $\mathbf{v}^{\text {(max) }}$ (A) being the largest right singular vector of the matrix $\mathbf{A}$. The dimensions of the matrix $\mathbf{Q}^{\text {(iid) }}$ are determined by $N_{i}$ and $M_{u, i}$, i.e., $\mathbf{Q}^{\text {(iid) }} \in \mathbb{C}^{M_{u, i} \times N_{i}}$.

These quantization strategies are denoted as correlated RCDQ. Depending on the employed antenna combiner, the corresponding codebook is applied.

\subsubsection{Differential quantization}

The constructions presented thus far assume single shot quantization of channel directions, neglecting the time or frequency correlation between consecutive quantization instants. One way to exploit the correlation inherent in the wireless channel with memoryless quantization is to provide feedback for a fraction of the CSI and to use interpolation at the base station to estimate the remaining CSI. This approach is appropriate for exploiting frequency correlation between subcarriers in OFDM systems [29,30], but it is not so useful for temporal correlation, because the interpolated CSI is already outdated at the time it is available.

Differential feedback is a flexible approach for reducing feedback requirements and providing high resolution $[37,38]$. Further improvements are possible by means of predictive coding, where previously quantized CSI is employed to predict the current CSI and only the prediction error is quantized $[30,39]$. In this study, the adaptive predictive channel direction quantization (APCDQ) algorithm of [10] is employed, which is an extension of [30] to DASs with single antenna receivers. Because this algorithm automatically adapts to the statistics of the channel direction, it can also be employed in multi receive antenna systems with antenna combining.

An overview of the considered SU-MIMO and MUMIMO transceiver architectures is provided in Table 1.

\section{Simulations}

This section presents cell throughput simulation results of a cellular network employing different distributed antenna configurations. The results are obtained with an extended version of the Vienna LTE-A link level simulator $[11,12]$. The simulator is augmented with a path loss model and extended for an accurate interference model, as detailed below.

Throughput results with perfect CSIT are presented, investigating the area spectral efficiency (ASE) obtained with different DAS configurations, and employing the SU-MIMO and MU-MIMO transmission strategies presented in Section 2.2. The efficiency of the CSI feedback algorithms of Section 3 is compared by means of ecdfs of the cell throughput. Also, a cross-comparison of centralized and distributed SU-MIMO and MUMIMO systems with quantized CSIT, in terms of ASE, is conducted.

The considered transmission methods, CSI feedback algorithms, path loss model, and out-of-cell interference model are part of version 1.1. of the Vienna LTE-A link-level simulator, facilitating reproducibility of the presented results.

\subsection{Simulation methodology}

When simulating DASs it is important to consider multiple cells, because the out-of-cell interference environment is impacted by the RRUs. System-level simulations of multi-cell DASs suffer from high computational complexity, because the described transmission and feedback strategies require knowledge of the physical details of the wireless channel.

In this article, a compromise simulation methodology is considered, which combines the accuracy of link-level simulations with the efficiency of abstracted system-level simulations. Thereby detailed link-level simulations are conducted for a single cell only, cell $i=$ 0 in Figure 1, whereas out-of-cell interference is taken

Table 1 Overview of the considered transceiver architectures

\begin{tabular}{llll}
\hline & TX precoding & RX combining & RX feedback \\
\hline \multirow{2}{*}{ SU-MIMO } & \multicolumn{2}{c}{ SVD - transceivers } & Perfect \\
& LTE codebook & ZF & LTE CLSM \\
MU-MIMO & ZF-beamforming & MET & Perfect \\
& ZF-beamforming & MET, QBC & RCDQ (corr.), \\
& & & APCDQ \\
\hline
\end{tabular}


into account employing the interference model proposed in [6]. In [6], the out-of-cell interference power per receive antenna for a system employing ZF MUMIMO is approximated using a second-order match to a Gamma distribution. The model assumes that the individual precoding vectors of different users, applied in the other cells, are independent and isotropically distributed, to simplify the analysis. In our simulations, we assume that all out-of-cell RRUs transmit with the same power, and that the maximum number of users is served in the interfering cells, $U_{j}=N_{j}, \forall j \neq 0$. The same model can also be used for SU-MIMO with small modifications, if the precoding matrices of users are assumed semi-unitary and isotropically distributed on $\mathcal{G}\left(N_{j}, \ell_{j}\right)$. Therefore, power loading is not considered with this model, and neither is the LTE codebook construction. In our SU-MIMO simulations, we assume transmission of the maximum number of streams in the other cells, i.e., $\ell_{j}=M_{k, j}, \forall j \neq i$ and $M_{k, j}=$ $M_{u, i}, \forall k, j$.

To emulate the out-of-cell interference, each received symbol is disturbed with a random complex-valued interference term whose power is distributed according to the derived Gamma distribution. The method significantly reduces simulation complexity, but on the downside it does not incorporate potential coupling in the scheduling decisions between cells. The detailed parameters of the Gamma distributions can be found in the Appendix.

\subsection{Simulation parameters and settings}

The simulation scenario under consideration in this section is shown in Figure 1. The simulated cellular network is compliant to the LTE-A specifications [16]. The total transmit power in each cell is $43 \mathrm{dBm}$ and a noise power spectral density of $-174 \mathrm{dBm} / \mathrm{Hz}$ is assumed. The network consists of 19 cells, arranged in a regular hexagonal grid, with a cell radius of $r_{c}=500 \mathrm{~m}$. Simulation results are presented for the center cell $i=0$, following the simulation methodology explained above.

Each cell contains $R_{i} \in\{0,3,4,6\}$ distributed antenna arrays that are equiangular spaced on a ring of radius $\frac{2}{3} r_{c}$, similar to [6]. Figure 1 shows the case for $R_{i}=6$. In practical situations, the placement of RRUs has to be carefully conducted, such as to optimize, e.g., the network coverage with respect to the large-scale shadow-fading realization in each cell. In our simulations, this complication is avoided by considering only path loss effects and not shadow-fading.

A total of $U_{i}=8$ users is served in each cell. Scheduling decisions are based on the proportional fairness metric according to Equation (1). In the SU-MIMO case, the scheduling framework presented in [40] is utilized, and for MU-MIMO the greedy scheduler ([31], Algorithm 2) is employed. In both cases, the achievable user throughput required for the scheduling decision, $R_{u}$ in (1) is estimated from the corresponding channel quality feedback, by mapping the SINR to spectral efficiency, via pre-computed LTE specific mapping tables (see [40] for details). For SU-MIMO, the CQI is estimated according to Section 3.1.2. For MU-MIMO, the SINR lower bound of Section 3.2.2 is employed for scheduling, and afterwards the CQI correction mentioned in Section 3.2.2 is applied to the scheduled users for transmission rate adaptation.

To speed-up the simulations, the smallest LTE specified system bandwidth of $B_{w}=1.4 \mathrm{MHz}$ is considered. The same reason forces us to employ a frequencyflat Rayleigh fading model for the small-scale channel matrix $\overline{\mathbf{H}}_{u, i}$ of (7), because then the same precoder is applicable for the total bandwidth, and CSI feedback for a single subcarrier only is sufficient. Instantaneous, non-causal CSI feedback is assumed, with a CSI feedback period of $1 \mathrm{~ms}$. This means that the CSI feedback is available already before the actual transmission. For the impact of frequency-selectivity and a feedback delay see, e.g., [30].

The temporal evolution of $\overline{\mathbf{H}}_{u, i}$ is determined by a correlated block-fading model. Thus, the channel is assumed constant during each LTE subframe of $1 \mathrm{~ms}$ duration, and channel realizations of consecutive subframes are correlated according to the maximum Doppler frequency $f_{d}$ [41]. The macro-scale path loss $L_{u, i}^{(n, j)}$ between user $u$ in cell $i$ and antenna $n$ in cell $j$, and thus the element $\gamma_{u, i}^{(n, j)}$ of the channel gain matrix (8), is determined by the distance $d_{u, i}^{(n, j)}$ according to

$$
\begin{aligned}
L_{u, i}^{(n, j)} & =\max \left(70,128.1+37.6 \log _{10}\left(\frac{d_{u, i}^{(n, j)}}{1000 \mathrm{~m}}\right)\right), \\
\gamma_{u, i}^{(n, j)} & =10^{-L_{u, i}^{(n, j)} / 10} .
\end{aligned}
$$

The total number of transmit antennas per cell is $N_{i}=8$, while different values for the number of receive antennas are considered from the set $M_{u, i} \in\{1,2,4\}$. The spatial correlation of $\overline{\mathbf{H}}_{u, i}$ is determined by a Kronecker correlation model [42]

$$
\overline{\mathbf{H}}_{u, i}=\mathbf{R}_{\mathrm{RX}}^{1 / 2} \overline{\overline{\mathbf{H}}}_{u, i} \mathbf{R}_{\mathrm{TX}}^{1 / 2}
$$

Here, $\mathbf{R}_{\mathrm{RX}}=\mathbb{E}\left(\overline{\mathbf{H}}_{u, i} \overline{\mathbf{H}}_{u, i}^{\mathrm{H}}\right)$ and $\mathbf{R}_{\mathrm{TX}}=\mathbb{E}\left(\overline{\mathbf{H}}_{u, i}^{\mathrm{H}} \overline{\mathbf{H}}_{u, i}\right)$ determine the correlation between the elements of $\overline{\mathbf{H}}_{u, i}$, and $\overline{\overline{\mathbf{H}}}_{u, i}$ is and i.i.d. matrix whose entries are uncorrelated. The channels between different receive antennas are assumed weakly correlated, which means that the 
off-diagonal elements of $\mathbf{R}_{\mathrm{RX}}$ are set equal to 0.3 . The transmit antennas are assumed uncorrelated, i.e., $\mathbf{R}_{\mathrm{TX}}=\mathbf{I}$. Note that a weak correlation between the channels to different antennas is advantageous for SU-MIMO systems [43], while the capacity of MU-MIMO systems with single-stream transmission per user increases with the antenna correlation [44].

The CSI feedback overhead is determined by the precoder codebook size and the accuracy of channel quality quantization. The CQI overhead for all feedback schemes is the same. In SU-MIMO, CLSM transmission, the LTEA specified precoder codebook of size 256 is employed, which amounts to a feedback rate of $8 \mathrm{bit} / \mathrm{ms} / \mathrm{user}$. If antenna subset selection is employed, two additional feedback bits are required. But, if less than eight transmit antennas are used, also the smaller LTE compliant codebooks are employed (4 bit codebook for four antennas, 2 bit for two antennas). Thus, the total average feedback rate of CLSM and CLSM with transmit antenna subset selection is almost equal; it even tends to decrease with antenna subset selection in DASs.

To achieve the same feedback overhead in the MUMIMO system, the codebook size of RCDQ is set to 256 as well. APCDQ employs a codebook with only 128 entries, because it requires one additional feedback bit for adaptation of the codebook. The simulation results are averaged over random codebook realizations.

Monte Carlo simulations are performed, in which the $U_{i}=8$ users per cell are uniformly distributed over the cell area. In total, 2,000 positions are generated for each user. The average cell throughput per position is estimated from the sample average over 200 small-scale fading and noise realizations. To provide a holistic metric of performance, the ASE [45] is employed. It is estimated using the discrete approximation

$$
\mathrm{ASE}=\frac{U_{i}}{r_{c}^{2} \pi} \sum_{k} T_{u}\left(r_{k}\right) \pi\left(r_{k}^{2}-r_{k-1}^{2}\right)
$$

where $T_{u}\left(r_{k}\right)$ denotes the average user throughput at a distance to the central base station between $r_{k-1}$ and $r_{k}$. A distance increment of $\Delta r_{k}=r_{k}-r_{k-1}=10 \mathrm{~m}$ is employed.

\subsection{Simulation results with perfect CSIT}

When perfect CSI is available at the base station, the SVD based scheme of Section 2.2.1 is employed for single-user transmission, and ZF beamforming with MET receive antenna combining, see Section 2.2.2, for MU-MIMO. The simulation results obtained for these two scenarios are shown in Figure 2, with different DAS configurations and varying numbers of receive antennas. The notation employed to identify a DAS configuration is explained using an example:

$$
N_{0, i}-N_{r, i} / R_{i}=2-1 / 6
$$

means that the number of transmit antennas at the antenna array of the central base station is $2, N_{0, i}=2$, the size of the antenna arrays employed at the RRUs is 1 , $N_{r, i}=1$, and a total of $R_{i}=6$ distributed antenna arrays

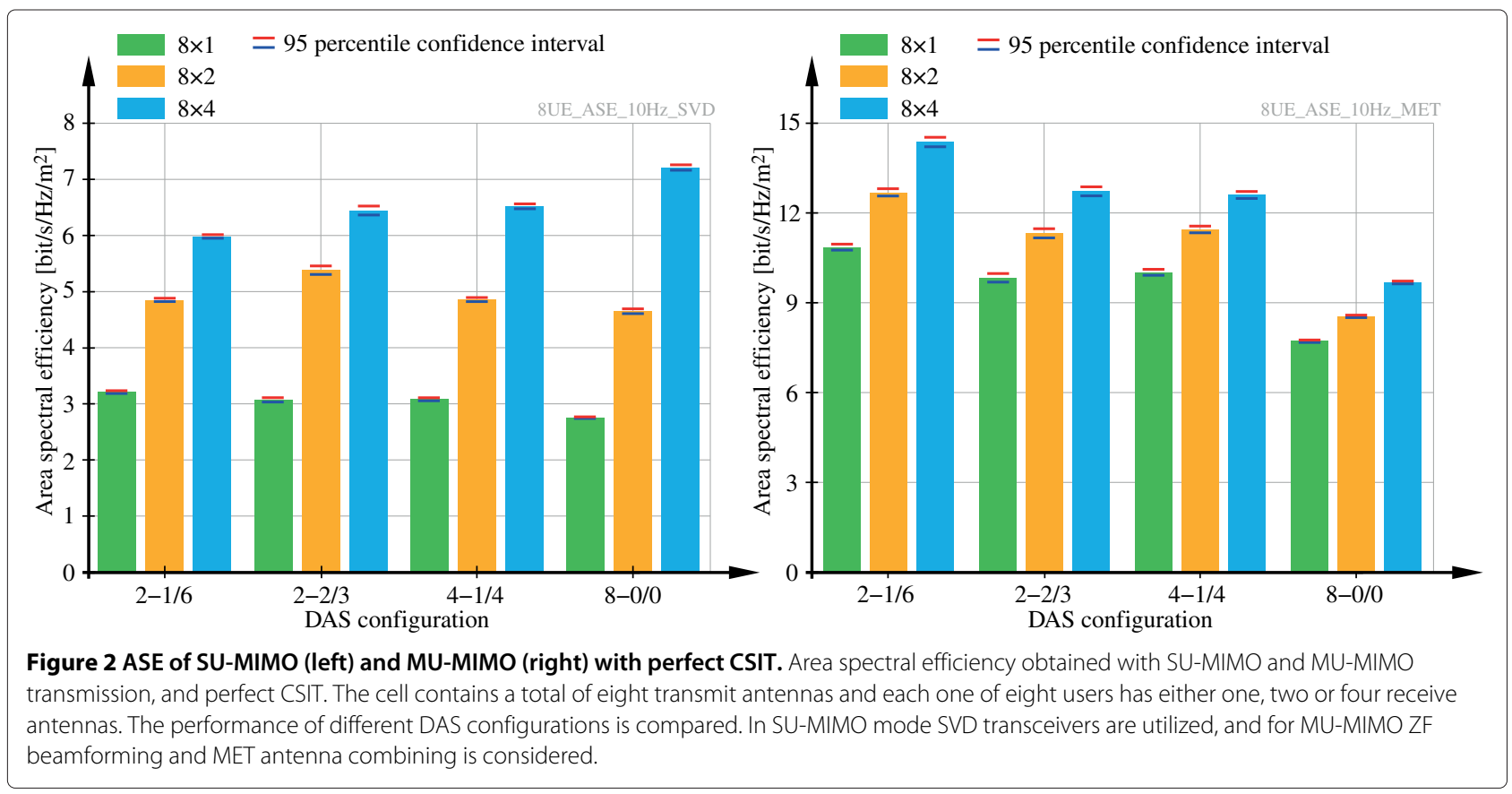


is employed in the cell. The total number of transmit antennas $N_{i}$ equals eight in all considered cases.

The left-hand side diagram of Figure 2 shows the ASE achieved with single-user transmission. It is observed, that the achieved ASE in the SU-MIMO system only shows a weak dependence on the employed DAS configuration. For $M_{u, i} \in\{1,2\}$ receive antennas, the best performance is achieved if the size of the antenna arrays at the RRUs matches the number of receive antennas. When $M_{u, i}=4$ is used, $N_{r, i}$ can only be matched to $M_{u, i}$ for a single antenna array, with the chosen parameters $N_{i}=8$ and $R_{i} \in\{0,3,4,6\}$. In this case, the best performance is achieved with the centralized system with $R_{i}=0$.

In the MU-MIMO system, shown in the right-hand side diagram of Figure 2, the performance improves with the number of RRUs available, independent of $M_{u, i}$. The $N_{0, i}-$ $N_{r, i} / R_{i}=2-1 / 6$ system gains approximately $45 \%$ in ASE compared to the $8-0 / 0$ configuration.

A cross-comparison of the left-hand side and right-hand side diagrams of Figure 2 reveals the large potential of MU-MIMO versus SU-MIMO especially if few receive antennas are available, a situation that is likely to happen in practice due to hardware complexity and space limitations encountered in handheld devices. The $2-2 / 3$ MU-MIMO system, e.g., triples the ASE of the SU-MIMO system with $M_{u, i}=1$, and doubles it for $M_{u, i} \in\{2,4\}$. The reason for this behavior is that the multiplexing gain in the SU-MIMO system is confined by the number of receive antennas, while the MU-MIMO system can achieve an $N_{i}$-fold multiplexing gain even if each user has just one antenna, by serving several users in parallel. Adding more receive antennas in the SU-MIMO system, the multiplexing gain can also be increased, but in practice the channel correlation between the receive antennas will confine the achievable multiplexing gain (remember that we assume a correlation coefficient of only 0.3 in our simulations, which is strongly idealistic for, e.g., handheld devices). Furthermore, the full multiplexing gain in SUMIMO systems is only achieved at very high SNR; see [24].

\subsection{Simulation results with limited feedback}

Here the impact of quantized CSIT is investigated. Only the centralized $N_{0, i}-N_{r, i} / R_{i}=8-0 / 0$ configuration and the $2-2 / 3$ DAS are considered in this section. Because $M_{u, i}=2$ is a likely option for most mobiles in practice, the $2-2 / 3$ DAS was chosen as it achieves the best SU-MIMO performance.

Figure 3 shows ecdfs of the SU-MIMO and MU-MIMO cell throughput achieved with the feedback algorithms detailed in Section 3, in comparison to the ones obtained with perfect CSIT. The results are shown for the $8-$ $0 / 0$ system, and a maximum Doppler frequency of $f_{d}=$ $10 \mathrm{~Hz}$, corresponding to a walking speed of approximately $5 \mathrm{~km} / \mathrm{h}$. The left-hand side diagram of Figure 3 shows the performance of SU-MIMO. It can be seen that CLSM performs close to SVD precoding if a single receive antenna $M_{u, i}=1$ is employed, while the throughput loss distinctly grows when $M_{u, i}>1$. Antenna subset selection is

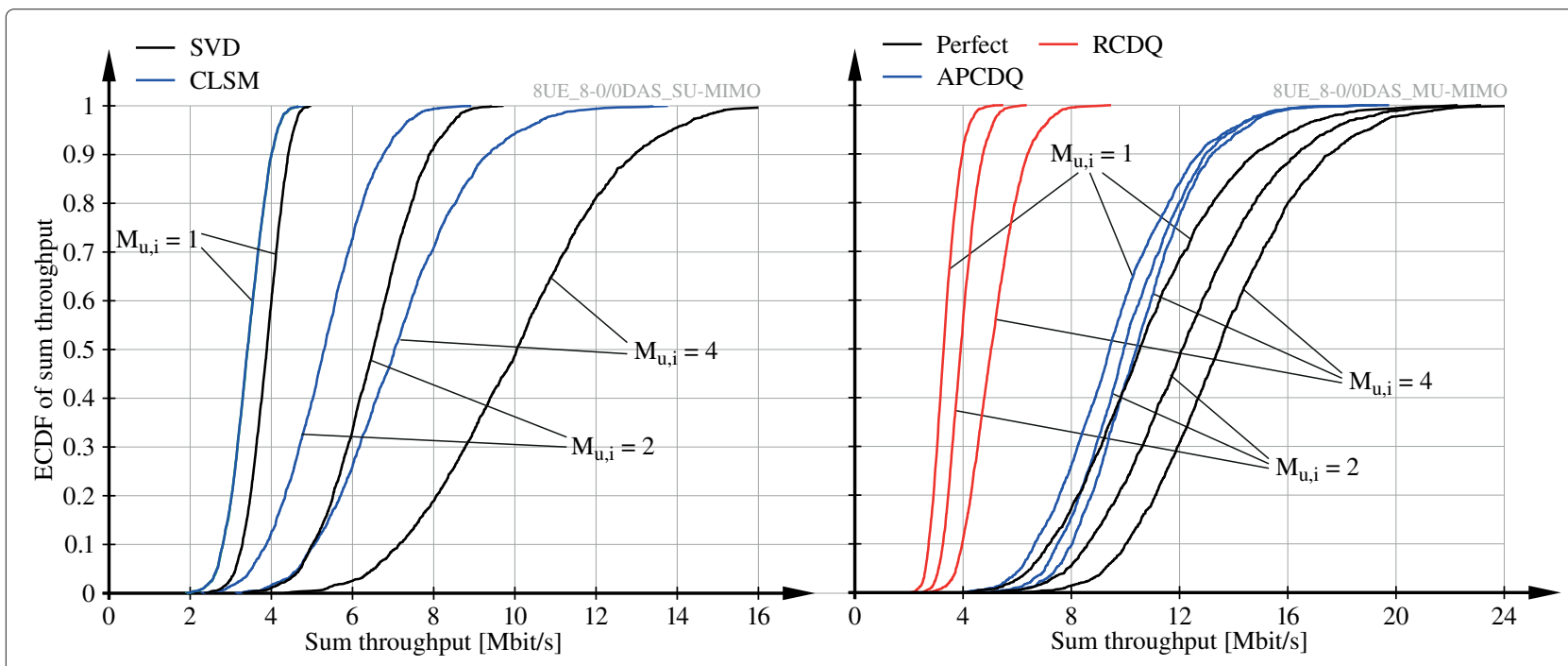

Figure 3 Cell throughput achieved with SU-MIMO (left) and MU-MIMO (right), without distributed antennas. Empirical cumulative density functions of the cell throughput obtained with SU-MIMO and MU-MIMO transmission in a cellular network without distributed antennas, and a total of eight transmit antennas. The performance with one, two, or four receive antennas per user is compared. The maximum Doppler frequency equals $f_{d}=10 \mathrm{~Hz}$. The SU-MIMO transmission is based on SVD precoding and equalization, or CLSM without antenna subset selection and ZF equalization. The MU-MIMO transmission is based on ZF precoding and MET or QBC antenna combining, with perfect and quantized CSIT. 
not considered in case of a centralized system, because all channel gains are equal.

The right-hand side diagram of Figure 3 shows ecdfs of the cell throughput achieved with MU-MIMO. Memoryless quantization, using QBC and the RCDQ feedback algorithm, is far from the possible performance achieved with perfect channel knowledge. ${ }^{\mathrm{c}}$ This is due to the residual in-cell interference caused by the quantization error. With multiple receive antennas, the throughput gap can be reduced, because the quantization-based combining algorithm reduces the quantization error.

When differential quantization is employed, utilizing the APCDQ algorithm, the performance loss can be markedly reduced. For $M_{u, i} \in\{1,2\}$ and at $f_{d}=10 \mathrm{~Hz}$, the APCDQ algorithm is employed in combination with MET. Here, the algorithm already provides accurate CSIT and it is better to exploit the channel gain improvement provided by MET instead of the quantization accuracy improvement of QBC. With $M_{u, i}=4$ receive antennas, it was observed that tracking the strongest eigenmode of the channel matrix utilizing APCDQ causes a larger error than tracking the channel direction of a single-receive antenna system. We believe the reason is that over time the magnitudes of the singular values of the channel matrix change, and thus the strongest eigenmode switches between different eigenmodes, causing the APCDQ algorithm to predict the effective channel direction wrongly. The increased quantization error is compensated by employing dominant subspace quantization-based combining, restricted to the $k=2$ dominant eigendimensions, instead of MET if $M_{u, i}=4$. At $f_{d}=50 \mathrm{~Hz}$, the best performance is obtained when combining APCDQ with QBC for $M_{u, i}=2$, and with dominant subspace $\mathrm{QBC}$ and $k=3$ for $M_{u, i}=4$.

The empirical cdfs of the distributed $2-2 / 3$ system are shown in Figure 4. Similar to the centralized system, there is a large throughput gap between CLSM based SUMIMO and the performance promised by SVD precoding if $M_{u, i}>1$, as shown in the left-hand side diagram of Figure 4. But, in contrast to the $8-0 / 0$ DAS, this gap can be strongly reduced by means of transmit antenna subset selection. A very similar, even larger gain, is possible by exploiting knowledge about the channel gains in the MUMIMO system, when employing correlated RCDQ instead of RCDQ; see the right-hand side diagram of Figure 4. The reason for this behavior is that in a DAS at most user positions large path loss differences between different RRUs exist. Thus, the DAS enables the users to mainly concentrate their feedback bits on the dominant set of antennas, because the RRUs with weak channel gains hardly contribute to the effective channel direction of a user. For the same reason also the problem of the APCDQ algorithm in tracking the strongest eigenmode vanishes, and MET is employed for all considered numbers of receive antennas $M_{u, i} \in\{1,2,4\}$.

A comparison of SU-MIMO employing CLSM with antenna subset selection, and MU-MIMO utilizing correlated RCDQ and APCDQ is shown in Figure 5, in terms of ASE. The memoryless quantization algorithms do not depend on the temporal correlation of the wireless

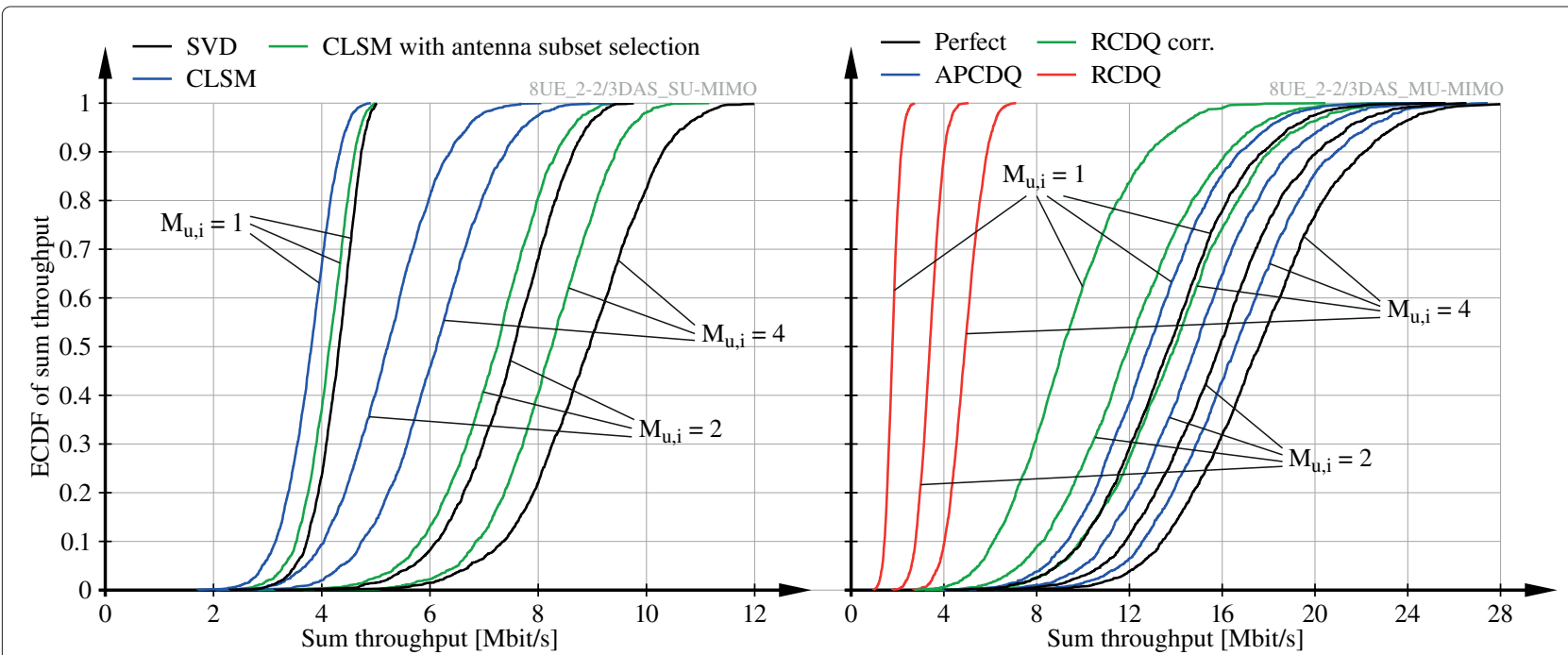

Figure 4 Cell throughput achieved with SU-MIMO (left) and MU-MIMO (right), with distributed antennas. Empirical cumulative density functions of the cell throughput obtained with SU-MIMO and MU-MIMO transmission in a cellular network with three distributed antenna arrays and one central antenna array, giving a total of eight transmit antennas. The performance with one, two, or four receive antennas per user is compared. The maximum Doppler frequency equals $f_{d}=10 \mathrm{~Hz}$. The SU-MIMO transmission is based on SVD precoding and equalization, or CLSM with/without antenna subset selection and ZF equalization. The MU-MIMO transmission is based on ZF precoding and MET or QBC receive antenna combining, with perfect and quantized CSIT. 


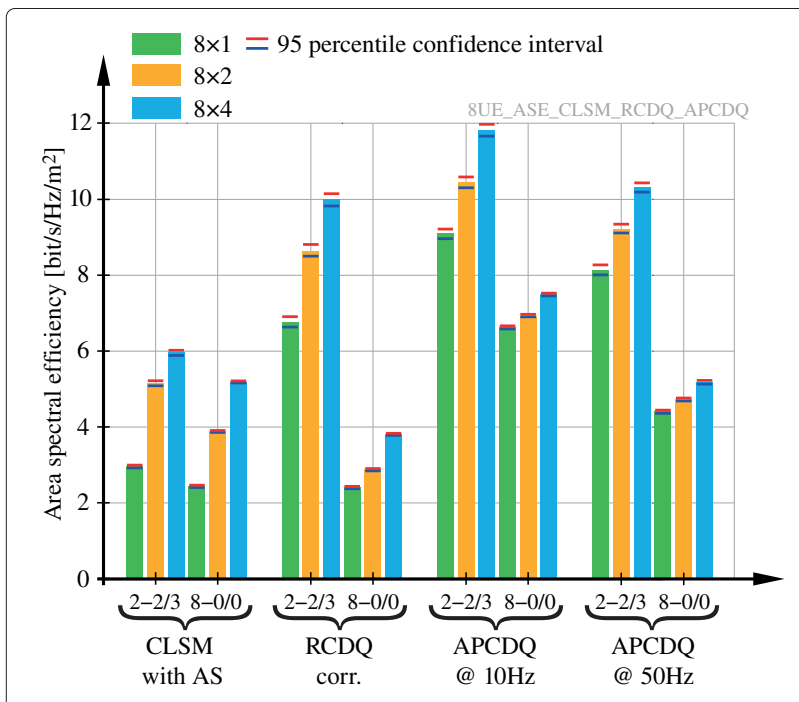

Figure 5 Comparison of the ASE achieved with SU-MIMO and MU-MIMO employing different CSI feedback algorithms. Area spectral efficiency obtained with SU-MIMO and MU-MIMO transmission utilizing the presented CSI feedback algorithms. The performance of the $2-2 / 3$ DAS configuration is compared to the one achieved in the centralized $8-0 / 0$ system.

channel, results are thus only shown for $f_{d}=10 \mathrm{~Hz}$. The APCDQ algorithm exploits temporal channel correlation to improve the quantization accuracy. Therefore, the achieved throughput with APCDQ depends on the Doppler frequency as demonstrated in the figure, by contrasting ASEs at $f_{d}=10 \mathrm{~Hz}$ and $f_{d}=50 \mathrm{~Hz}$. Considering the results achieved with CLSM, it can be seen that the gain of employing distributed antennas depends on the number of receive antennas, but is only in the order of 15 to $30 \%$. For MU-MIMO, this gain is markedly increased; with memoryless quantization it is in the order of $150 \%$, while differential quantization performs around $75 \%$ better with distributed antennas.

A cross-comparison of single-user and multi-user spatial multiplexing shows that, with the considered quantization codebook size and memoryless quantization, MU-MIMO is only advantageous in the DAS and not in the centralized system. But note that the potential performance improvement achieved by increasing the codebook size is larger for multi-user RCDQ than for single-user CLSM, because the gap to perfect channel knowledge is larger (see Figure 3).

With APCDQ, the throughput of the MU-MIMO system is improved, especially in low-mobility scenarios, such that it always outperforms SU-MIMO at the considered Doppler frequencies. When $f_{d}=50 \mathrm{~Hz}$, which corresponds to a speed of $25 \mathrm{~km} / \mathrm{h}$, the gain of APCDQ compared to CLSM in the centralized system with four receive antennas drops almost to zero. Thus, with moderate feedback overhead the scope of MUMIMO is confined to low to moderate mobility scenarios.

As a final remark it should be mentioned that the presented results favor MU-MIMO, because a CSI feedback delay of zero is assumed. The sensitivity with respect to out-dated feedback is clearly larger for MU-MIMO than for SU-MIMO. Still, in low to moderate mobility scenarios, a reasonable feedback delay can be compensated by prediction algorithms at the receivers [30], thus justifying the validity of the presented results.

\section{Conclusion}

In this article, cellular networks employing distributed antennas are investigated by means of LTE standard compliant Şhybrid link/system level simulations. The throughput performance of distributed antenna systems and centralized systems, having all available transmit antennas collocated at the central base station, is compared. The work contrasts single-user spatial multiplexing, where multiple data streams are sent in parallel to a single receiver, and multi-user spatial multiplexing, which serves multiple users in parallel with a single stream per user, utilizing the MIMO capabilities of the system. Different receive antenna combining techniques are considered for MU-MIMO.

Several approaches for limited feedback applied to distributed antennas are analyzed in this study as well. The impact of exploiting side information on the average largescale fading is considered, and shown to considerably improve the CSI quantizer performance.

With perfect channel state information at the base station, it turns out that SU-MIMO only gains from distributing antennas over the cell area, if the number of receive antennas is less than or equal to the number of antennas at the RRUs. In this case, the macroscopic diversity gain provided by the DAS can be fully exploited. On the other hand, if the antenna array at the receiver is larger than those used at the RRUs, the DAS can often not support the multiplexing capabilities of the receiver. Then better performance is achieved if all available antennas are collocated at the central base station. With quantized channel state information only being available at the base station, the situation changes in favor of the DAS. Utilizing the LTE-A-specified precoder codebook together with a simple transmit antenna subset selection algorithm, gains in the order of $20 \%$ in terms of ASE are observed.

When the system employs MU-MIMO with single stream transmission per user, the DAS clearly outperforms the centralized system, if perfect CSIT is available, due to the improved macro-diversity. If side-information about the path loss with respect to the individual RRUs is available at the base station, the same holds true with quantized CSIT. 
A cross-comparison of single-user and multi-user transmission demonstrates a large ASE gain of MU-MIMO, if sufficiently accurate CSIT is available. The required CSIT accuracy is easier to obtain in the DAS, if side-information about large-scale fading is exploited.

\section{Endnotes}

${ }^{\mathrm{a}} \mathrm{A} p \times q$ matrix $\mathbf{U}$ with $q \leq p$ is called semi-unitary if $\mathbf{U}^{\mathrm{H}} \mathbf{U}=\mathbf{I}_{q}$.

${ }^{\mathrm{b}}$ Note the different definitions of our channel matrices $\mathbf{H}_{u, i}$ and those used by Jindal [23]. Our matrix equals the conjugate-transpose of their definition.

${ }^{\mathrm{c}} \mathrm{RCDQ}$ and correlated RCDQ are equal in the centralized system, because the channel gains with respect to all antennas are equal.

\section{Appendix: Out-of-cell interference model}

In this section, we provide the detailed parameters of the Gamma distribution model for the out-of-cell interference, according to [6].

\section{MU-MIMO}

The out-of-cell interference is generated for each receive antenna individually. Hence, we consider the vector channels determined by the individual rows of the matrix $\mathbf{H}_{u, i}^{(j)}$. We denote an arbitrary row of $\mathbf{H}_{u, i}^{(j)}$ as $\mathbf{h}_{u, i}^{(j)} \mathrm{H}$. The received signal on the considered antenna before the receiver antenna combiner is thus given by

$$
r_{u, i}=\mathbf{h}_{u, i}^{\mathrm{H}} \mathbf{F}_{u, i} \mathbf{x}_{u, i}+\mathbf{h}_{u, i}^{\mathrm{H}} \sum_{k \neq u}^{U_{i}} \mathbf{F}_{k, i} \mathbf{x}_{k, i}+\sum_{j \neq i}^{I} \mathbf{h}_{u, i}^{(j)}{ }^{\mathrm{H}} \mathbf{F}_{j} \mathbf{x}_{j}+z_{u, i} .
$$

The out-of-cell Gamma distribution model of [6] is based on matching the first- and second-order moments of the out-of-cell interference power to a Gamma distribution. We thus have to compute these moments first. The instantaneous out-of-cell interference power is given by

$$
I_{u, i}=\sum_{j \neq i}^{I}\left\|\mathbf{h}_{u, i}^{(j)} \mathrm{H}_{j} \mathbf{F}_{j}\right\|^{2}=\sum_{j \neq i}^{I} \sum_{k=1}^{U_{j}}\left|\mathbf{h}_{u, i}^{(j)} \mathrm{H}_{\mathbf{f}_{k, j}}\right|^{2} .
$$

According to ([6], Proposition 11), the first- and secondorder moments of $\left|\mathbf{h}_{u, i}^{(j)} \mathrm{H}_{\mathbf{f}_{k, j}}\right|^{2}$ are given by (note that there is a typo in ([6], Proposition 11)

$$
\begin{aligned}
& \mu_{u, i}^{(j)}=\frac{1}{N_{j}} \sum_{n=1}^{N_{j}} \frac{P_{j}}{U_{j}} \gamma_{u, i}^{(n, j)}=\frac{1}{N_{j}} \sum_{n=1}^{N_{j}} \sigma_{u, i}^{(n, j)^{2}}, \\
& {\sigma_{u, i}^{(j)}}^{2}=\frac{N_{j}-1}{N_{j}^{2}\left(N_{j}+1\right)}\left(2 \sum_{n=1}^{N_{j}} \sigma_{u, i}^{(n, j)^{4}}+\sum_{n=1}^{N_{j}} \sum_{l \neq n} \sigma_{u, i}^{(n, j)^{2}} \sigma_{u, i}^{(l, j)^{2}}\right) .
\end{aligned}
$$

The Gamma approximation of one interference term according to ([6], Lemma 7) equals

$$
\begin{gathered}
\left|\mathbf{h}_{u, i}^{(j)} \mathrm{H}_{k, j}\right|^{2} \sim \Gamma\left(k_{u, i}^{(j)}, \theta_{u, i}^{(j)}\right), \\
k_{u, i}^{(j)}=\frac{\mu_{u, i}^{(j)}{ }^{2}}{\sigma_{u, i}^{(j)^{2}}}, \quad \theta_{u, i}^{(j)}=\frac{\sigma_{u, i}^{(j)^{2}}}{\mu_{u, i}^{(j)}} .
\end{gathered}
$$

Using Proposition 8 in [6], the sum interference in (62) can be matched with yet another Gamma random variable, whose parameters are given by

$$
\begin{gathered}
I_{u, i} \sim \Gamma\left(k_{u, i}, \theta_{u, i}\right), \\
k_{u, i}=\frac{\left(\sum_{j \neq i} U_{j} k_{u, i}^{(j)} \theta_{u, i}^{(j)}\right)^{2}}{\sum_{j \neq i} U_{j} k_{u, i}^{(j)} \theta_{u, i}^{(j)}{ }^{2}}, \theta_{u, i}=\frac{\sum_{j \neq i} U_{j} k_{u, i}^{(j)} \theta_{u, i}^{(j)}{ }^{2}}{\sum_{j \neq i} U_{j} k_{u, i}^{(j)} \theta_{u, i}^{(j)}} .
\end{gathered}
$$

In our simulations wet set $U_{j}=N_{j}, \forall j \neq i$ and $P_{j}=P, \forall j$.

\section{SU-MIMO}

With SU-MIMO, the out-of-cell interference power is given by

$$
I_{u, i}=\sum_{j \neq i}^{I}\left\|\mathbf{h}_{u, i}^{(j)} \mathrm{H}_{j}\right\|^{2}=\sum_{j \neq i}^{I} \sum_{\ell=1}^{\ell_{j}}\left|\mathbf{h}_{u, i}^{(j)} \mathrm{H}_{\mathbf{f}_{\ell, j}}\right|^{2} .
$$

The difference to MU-MIMO is that the second sum above now goes over the streams of a single user and not over the streams of different users. Hence, also the precoder $\mathbf{F}_{j} \in \mathbb{C}^{N_{j} \times \ell_{j}}$ is different. It cannot be assumed that the columns of $\mathbf{F}_{j}$ are independent and isotropically distributed as in [6], but the precoding matrix is semi-unitary, i.e.,

$$
\mathbf{F}_{j}^{\mathrm{H}} \mathbf{F}_{j}=\frac{P_{j}}{\ell_{j}} \mathbf{I} .
$$

For a fixed channel $\mathbf{h}_{u, i}^{(j)}$ and assuming $\mathbf{F}_{j}$ to be isotropically distributed on $\mathcal{G}\left(N_{j}, \ell_{j}\right)$, it is know that the term $\frac{\ell_{j}}{P_{j}} \frac{\left\|\mathbf{F}_{j}^{\mathrm{H}} \mathbf{h}_{u, i}^{(j)}\right\|^{2}}{\left\|\mathbf{h}_{u, i}^{(j)}\right\|^{2}}$ is beta distributed with parameters $\beta\left(\ell_{j}, N_{j}-\ell_{j}\right)$ [46] (after generalization to the complexvalued case). Using the properties of a beta distribution, the conditional mean and variance of one interference term are obtained as

$$
\begin{aligned}
\mu_{\mid \mathbf{h}_{u, i}^{(j)}} & :=\mathbb{E}\left(\left\|\mathbf{h}_{u, i}^{(j)} \mathrm{H}_{j}\right\|^{2} \mid \mathbf{h}_{u, i}^{(j)}\right) \\
& =\frac{P_{j}}{\ell_{j}}\left\|\mathbf{h}_{u, i}^{(j)}\right\|^{2} \mathbb{E}\left(\beta\left(\ell_{j}, N_{j}-\ell_{j}\right)\right) \\
& =\frac{P_{j}}{\ell_{j}}\left\|\mathbf{h}_{u, i}^{(j)}\right\|^{2} \frac{\ell_{j}}{N_{j}}=\frac{P_{j}}{N_{j}}\left\|\mathbf{h}_{u, i}^{(j)}\right\|^{2} .
\end{aligned}
$$




$$
\begin{aligned}
\operatorname{var}_{\mid \mathbf{h}_{u, i}^{(j)}} & :=\operatorname{var}\left(\left\|\mathbf{h}_{u, i}^{(j)} \mathrm{H} \mathbf{F}_{j}\right\|^{2} \mid \mathbf{h}_{u, i}^{(j)}\right) \\
& =\left(\frac{P_{j}}{\ell_{j}}\right)^{2}\left\|\mathbf{h}_{u, i}^{(j)}\right\|^{4} \frac{\ell_{j}\left(N_{j}-\ell_{j}\right)}{N_{j}^{2}\left(N_{j}+1\right)} \\
& =\frac{N_{j}-\ell_{j}}{\ell_{j}\left(N_{j}+1\right)} \mu_{\mid \mathbf{h}_{u, i}^{(j)}}^{2} .
\end{aligned}
$$

Using ([6], Proposition 11), the conditioning on $\mathbf{h}_{u, i}^{(j)}$ can be removed, leading to the moments

$$
\begin{aligned}
\mu_{u, i}^{(j)}= & \frac{1}{N_{j}} \sum_{n=1}^{N_{j}} P_{j} \gamma_{u, i}^{(n, j)}=\frac{1}{N_{j}} \sum_{n=1}^{N_{j}} \sigma_{u, i}^{(n, j)^{2}}, \\
\sigma_{u, i}^{(j) 2}= & \frac{N_{j}-\ell_{j}}{N_{j}^{2} \ell_{j}\left(N_{j}+1\right)} \\
& \times\left(2 \sum_{n=1}^{N_{j}} \sigma_{u, i}^{(n, j)^{4}}+\sum_{n=1}^{N_{j}} \sum_{l \neq n} \sigma_{u, i}^{(n, j)^{2}} \sigma_{u, i}^{(l, j)^{2}}\right) .
\end{aligned}
$$

The difference compared to MU-MIMO is a missing factor of $1 / U_{j}$ in $\sigma_{u, i}^{(n, j)^{2}}$, and the different pre-factor in $\sigma_{u, i}^{(j)^{2}}$. With ([6], Proposition 8) we obtain the Gamma match for the sum interference

$$
\begin{aligned}
I_{u, i} & \sim \Gamma\left(k_{u, i}, \theta_{u, i}\right), \\
k_{u, i} & =\frac{\left(\sum_{j \neq i} k_{u, i}^{(j)} \theta_{u, i}^{(j)}\right)^{2}}{\sum_{j \neq i} k_{u, i}^{(j)} \theta_{u, i}^{(j)}{ }^{2}}, \theta_{u, i}=\frac{\sum_{j \neq i} k_{u, i}^{(j)} \theta_{u, i}^{(j)^{2}}}{\sum_{j \neq i} k_{u, i}^{(j)} \theta_{u, i}^{(j)}}, \\
k_{u, i}^{(j)} & =\frac{\mu_{u, i}^{(j)}{ }^{2}}{\sigma_{u, i}^{(j)}}, \quad \theta_{u, i}^{(j)}=\frac{\sigma_{u, i}^{(j)^{2}}}{\mu_{u, i}^{(j)}} .
\end{aligned}
$$

\section{Abbreviations}

APCDQ: Adaptive predictive channel direction quantization; ASE: Area spectral efficiency; AWGN: Additive white gaussian noise; BICM: Bit interleaved coded modulation; CDI: Channel direction indicator; CLSM: Closed loop spatial multiplexing; CQI: Channel quality indicator; CSI: Channel state information; CSIT: Channel state information at the transmitter; DAS: Distributed antenna system; ECDF:[ecdf]Empirical cumulative density function; lid:[i.i.d.]Independent identically distributed; LTE: Long Term Evolution; LTE-A: Long Term Evolution Advanced; MCS: Modulation and coding scheme; MET: Multi-user eigenmode transmission; MIESM: Mutual information effective SINR mapping; MSE: Mean squared error; MU-MIMO: Multi-user MIMO; PMI: Precoding matrix indicator; QBC: Quantization-based combining; RCDQ: Random channel direction quantization; RI: Rank indicator; RRU: Remote radio unit; SINR: Signal to interference and noise ratio; SNR: Signal to noise ratio; SU-MIMO: Single-user MIMO; SVD: Singular value decomposition; TTI: Transmission time interval; ZF: Zero forcing.

\section{Competing interests}

The authors declare that they have no competing interests.

\section{Acknowledgements}

This study was funded by A1 Telekom Austria AG and the Institute of Telecommunications, Vienna University of Technology. The authors wish to thank the Institute of Telecommunications' LTE research group for continuous support. This material is based upon work supported by the National Science Foundation under Grant nos. 0830615 and 1218338.

\section{Author details}

${ }^{1}$ Institute of Telecommunications, Vienna University of Technology, Vienna, Austria. ${ }^{2}$ Department of Electrical and Computer Engineering, The University of Texas at Austin, Austin, TX, USA.

\section{Received: 20 November 2012 Accepted: 20 February 2013} Published: 19 March 2013

\section{References}

1. M Riaz, R Nielsen, J Pedersen, N Prasad, O Madsen, in International Conference on Wireless and Optical Communications Networks. On radio over fiber for heterogeneous wireless networks (Cairo Egypt, 2009), pp. 379-382

2. A Saleh, A Rustako, R Roman, Distributed antennas for indoor radio communications. IEEE Trans. Commun. 35(12), 1245-1251 (1987)

3. P Chow, A Karim, V Fung, C Dietrich, in IEEE 44th Vehicular Technology Conference, vol. 3. Performance advantages of distributed antennas in indoor wireless communication systems (Sweden Stockholm, 1994), pp. 1522-1526

4. K Kerpez, S Ariyavisitakul, in IEEE Global Telecommunications Conference, vol. 3. A radio access system with distributed antennas (San Francisco California, 1994), pp. 1696-1700

5. W Choi, J Andrews, Downlink performance and capacity of distributed antenna systems in a multicell environment. IEEE Trans. Wirel. Commun. 6, 69-73 (2007)

6. R Heath Jr, T Wu, YH Kwon, ACK Soong, Multiuser MIMO in distributed antenna systems with out-of-cell interference. IEEE Trans. Signal Process 59(10), 4885-4899 (2011)

7. W Roh, A Paulraj, in IEEE 57th Vehicular Technology Conference, vol. 1. Performance of the distributed antenna systems in a multi-cell environment (Jeju Island Korea, 2003), pp. 587-591

8. X Chen, Z Zhang, HH Chen, On distributed antenna systems with limited feedback precoding: opportunities and challenges. IEEE Wirel. Commun. 17(2), 80-88 (2010)

9. E Zeng, S Zhu, Z Zhong. On the performance of adaptive limited feedback beamforming in distributed MIMO systems (New Orleans Louisiana, 2008), pp. 1-5

10. S Schwarz, R Heath Jr, M Rupp, in IEEE 4th Int. Workshop on Heterogeneous and Small Cell Networks, GLOBECOM. Multiuser MIMO in distributed antenna systems with limited feedback (Anaheim California, 2012), pp. 546-551

11. http://www.nt.tuwien.ac.at/ltesimulator/

12. CMehlführer, JC Ikuno, M Simko, S Schwarz, M Rupp, The Vienna LTE simulators - enabling reproducibility in wireless communications research. EURASIP J. Adv. Signal Process. 2011 Special Issue on Reproducible Research, 1-13 (2011)

13. F Kelly, Charging and rate control for elastic traffic. Eur. Trans. Telecommun. 8, 33-37 (1997)

14. D Tse, P Viswanath, Fundamentals of Wireless Communications (Cambridge University Press. (Cambridge, MA, 2008)

15. DJ Love, R Heath Jr, W Santipach, M Honig, What is the value of limited feedback for MIMO channels. IEEE Commun. Mag. 42, 54-59 (2004)

16. 3GPP, Techn. Spec. Group Radio Access Network; E-UTRA. Phy. Chan. and Mod. (Release 10) (2010). http://www.3gpp.org/ftp/Specs/html-info/ 36211.htm

17. 3GPP, Techn. Spec. Group Radio Access Network; E-UTRA, (2009). http:// www.3gpp.org/ftp/Specs/html-info/36211.htm

18. J Wang, M Wu, F Zheng, in 6th International Conference on Wireless Communications Networking and Mobile Computing. The codebook design for MIMO precoding systems in LTE and LTE-A (Chengdu China, 2010), pp. 1-4

19. X Yun, X She, L Chen, J Zhu, Y Jiang, H Taoka, K Kusume, S Nagata, in IEEE 73rd Vehicular Technology Conference. Investigation of optimum double codebook design for downlink MIMO in LTE-A (Budapest Hungary, 2011), pp. 1-5

20. D Lim, K Choi, H Liu, in Sixth International Conference on Information Technology, New Generations. Optimum power allocation for distributed antenna systems with large-scale fading-only feedback (Las Vegas Nevada, 2009), pp. 1158-1164 
21. A Bayesteh, AK Khandani, in Proceedings of the International Symposium on Information Theory. On the user selection in MIMO broadcast channels (Adelaide Australia, 2005), pp. 2325-2329

22. F Boccardi, H Huang, in IEEE International Conference on Acoustics, Speech and Signal Processing, vol. 3. A near-optimum technique using linear precoding for the MIMO broadcast channel (Honolulu Hawaii, 2007) pp. $17-20$

23. N Jindal, Antenna combining for the MIMO downlink channel. IEEE Trans. Wirel. Commun. 7(10), 3834-3844 (2008)

24. S Schwarz, M Rupp, in Signal Processing Advances in Wireless Communications. Throughput maximizing feedback for MIMO OFDM based wireless communication systems (San Francisco California, 2011), pp. $316-320$

25. R Love, D Heath Jr, T Strohmer, Grassmannian beamforming for multiple-input multiple-output wireless systems. IEEE Trans. Inf. Theory. 49(10), 2735-2747 (2003)

26. D Love, R Heath, Limited feedback unitary precoding for spatial multiplexing systems. IEEE Trans. Inf. Theory. 51(8), 2967-2976 (2005)

27. G Caire, G Taricco, E Biglieri, Bit-interleaved coded modulation. IEEE Trans. Inf. Theory. 44(3), 927-946 (1998)

28. M Wrulich, M Rupp, Computationally efficient MIMO HSDPA system-level modeling. EURASIP J. Wirel. Commun. Netw. 2009, 1-14 (2009)

29. J Choi, R Heath Jr, Interpolation based transmit beamforming for MIMO-OFDM with limited feedback. IEEE Trans. Signal Process. 53(11), 4125-4135 (2005)

30. S Schwarz, M Rupp, in IEEE International Conference on Communications. Adaptive channel direction quantization-enabling multi user MIMO gains in practice (Ottawa Canada, 2012), pp. 6947-6952

31. M Trivellato, F Boccardi, F Tosate, in 65th IEEE Vehicular Technology Conference. User selection schemes for MIMO broadcast channels with limited feedback (Dublin Ireland, 2007), pp. 2089-2093

32. JC Ikuno, M Wrulich, M Rupp, in International ITG Workshop on Smart Antennas (WSA 2009). Performance and modeling of LTE H-ARQ (Berlin Germany, 2009), pp. 1-6

33. B Mondal, S Dutta, R Heath Jr, Quantization on the Grassmann manifold. IEEE Trans. Signal Process. 55(8), 4208-4216 (2007)

34. CK Au-Yeung, DJ Love, On the performance of random vector quantization limited feedback beamforming in a MISO system. IEEE Trans. Wirel. Commun. 6(2), 458-462 (2007)

35. N Jindal, MIMO, broadcast channels with finite-rate feedback. IEEE Trans. Inf. Theory. 52(11), 5045-5060 (2006)

36. D Love, R Heath Jr, Limited feedback diversity techniques for correlated channels. IEEE Trans. Veh. Technol. 55(2), 718-722 (2006)

37. OE Ayach, R Heath Jr, Grassmannian differential limited feedback for interference alignment. CoRR (2011). abs/1111.4596

38. M Islam, R Adve, in IEEE 22nd International Symposium on Personal Indoor and Mobile Radio Communications. Adaptive differential feedback in time-varying multiuser MIMO channels (Toronto Canada, 2011), pp. 2055-2059

39. T Inoue, R Heath $\mathrm{Jr}$, in 47 th Annual Allerton Conference on Communication Control, and Computing. Grassmannian predictive coding for delayed limited feedback MIMO systems (Monticello Illinois, 2009), pp. 783-788

40. S Schwarz, C Mehlführer, M Rupp, in 44th Annual Asilomar Conference on Signals, Systems, and Computers. Low complexity approximate maximum throughput scheduling for LTE (Pacific Grove California, 2010), pp. 1563-1569

41. YR Zheng, C Xiao, Simulation models with correct statistical properties for Rayleigh fading channels. IEEE Trans. Commun. 51(6), 920-928 (2003)

42. C Oestges, in IEEE 63rd Vehicular Technology Conference, vol. 6. Validity of the Kronecker model for MIMO correlated channels (Melbourne Australia, 2006), pp. 2818-2822

43. DS Shiu, GJ Foschini, MJ Gans, JM Kahn, Fading correlation and its effect on the capacity of multielement antenna systems. IEEE Trans. Commun. 48(3), 502-513 (2000)
44. R Louie, M McKay, I Collings, B Vucetic, in IEEE International Conference on Communications. Capacity approximations for multiuser MIMO-MRC with antenna correlation (Glasgow Scotland, 2007), pp. 5195-5200

45. MS Alouini, A Goldsmith, in IEEE 47th Vehicular Technology Conference, vol. 2. Area spectral efficiency of cellular mobile radio systems (Phoenix Arizona, 1997), pp. 652-656

46. J Nielsen, The distribution of volume reductions induced by isotropic random projections. Adv. Appl. Probab. 31(4), 985-994 (1999)

doi:10.1186/1687-6180-2013-54

Cite this article as: Schwarz et al.: Single-user MIMO versus multi-user MIMO in distributed antenna systems with limited feedback. EURASIP Journal on Advances in Signal Processing 2013 2013:54.

\section{Submit your manuscript to a SpringerOpen ${ }^{\mathcal{O}}$ journal and benefit from:}

- Convenient online submission

Rigorous peer review

- Immediate publication on acceptance

- Open access: articles freely available online

- High visibility within the field

- Retaining the copyright to your article

Submit your next manuscript at $\gg$ springeropen.com 